\title{
The law of refraction and Kepler's heuristics
}

\section{Carlos Alberto Cardona Suárez ${ }^{1}\left[\right.$ [ · Juliana Gutiérrez Valderrama ${ }^{1}[$}

Received: 25 February 2019 / Published online: 31 August 2019

(c) The Author(s) 2019

\begin{abstract}
Johannes Kepler dedicated much of his work to discover a law for the refraction of light. Unfortunately, he formulated an incorrect law. Nevertheless, it was useful for anticipating the behavior of light in some specific conditions. Some believe that Kepler did not have the elements to formulate the law that was later accepted by the scientific community, that is, the Snell-Descartes law. However, in this paper, we propose a model that agrees with Kepler's heuristics and that is also successful in anticipating the behavior of light when it passes through a surface that separates two media with different optical densities. This model adopts strategies that were recommended by Kepler in two types of analogies. The obstacles that led to the failure of the two types of analogies are presented in the article, and we argue that the model proposed here could overcome these specific obstacles. Finally, we show how the proposed model could be articulated with Kepler's metaphysics of light.
\end{abstract}

\section{Introduction}

In 1600, Johannes Kepler joined Tycho Brahe, who by that time had become imperial mathematician in the court of Rudolf II. Tycho died in 1601 as a result of a bladder disease. ${ }^{1}$ The astronomer's relatives then entered a bitter dispute with the Emperor with regard to the ownership of the tools and data that had been gathered by Tycho throughout his life. This dispute forced Kepler, who had by then taken up the role of imperial mathematician, to rethink his research agenda. In a letter written to his friend Herwart von Hohenburg, Kepler wrote: "Because I have had my diligence called into doubt, I have assumed the obligation for two works. The one to be ready for Easter

\footnotetext{
1 Cfr. Caspar (1959/1993), p. 121 and p. 123.

Communicated by Alan Shapiro.

\uliana Gutiérrez Valderrama juliana.gutierrez@urosario.edu.co

Carlos Alberto Cardona Suárez carlos.cardona@urosario.edu.co

1 Universidad del Rosario, Calle 12c \#6-25, Bogotá, Colombia
} 
[1603] will be Commentaries on the Theory of Mars [...] The other, to be finished off within 8 weeks, is the Optical Part of Astronomy" [quoted in Voelkel (2001, p. 147)]. The first project produced the Astronomia nova and was completed in 1609; the second compiled the author's most important contributions to optics and was completed by 1604. This work was published under the title Ad Vitellionem Paralipomena, quibus Astronomiae pars optica traditur. ${ }^{2}$ Kepler acknowledged that only a precise law of refraction would enable correction of the distortions in astronomical observations that are produced by the refraction of the light rays when passing through the celestial ether to the Earth's air and then to the eye.

When a light ray passes from one medium to another, e.g., from air to water, the rays change their direction of propagation. The thinkers who had studied refraction had reiterated that if the second medium was denser than the first, any light ray would move closer to the normal; otherwise, it would move farther away from the normal. However, before the seventeenth century, no one had any clarity of what "optical density" meant. Everyone hoped that it had something to do with material density.

By the end of the sixteenth century and in the early part of the seventeenth century, there was an urgent demand to determine a quantitative law of refraction. This knowledge was required to improve the use of lenses in the construction of telescopes. In his works De refractione optices and De telescopio, Della Porta presented qualitative descriptions that could only be adopted by the scientific community if they were complemented by a precise law of refraction. The British scholar Thomas Harriot had apparently determined the law of sines by 1602, even before Kepler had dealt with these matters. ${ }^{3}$ Willebrord Snell then arrived at this law almost simultaneously with Descartes in 1620; the latter then published it years later in his Dioptrica. Fermat disagreed with Descartes and formulated the law of sines in terms of the resistance offered by the two media (Cfr. de Fermat 1891, p. 117). Huygens arrived at the same law in 1690 when using an undulatory interpretation of light (Cfr. Huygens 1690/1945, pp. 28-45). Prior to the publication of the law of the sines in the Discours de la méthode, Mersenne had already made it public in 1636 and he attributed it to Descartes.

In the fourth chapter of the Paralipomena, after criticizing some of the efforts made up to that stage to find a law of refraction (including Tycho's efforts), Kepler suggested a new tactical orientation that would guide him through the search for this law. This new reorientation was stated as follows:

For geometrical terms ought to be at our service for analogy. I love analogies most of all: they are my most faithful teachers, aware of all the hidden secrets of nature. In geometry in particular they are to be taken up, since they restrict the infinity of cases between their respective extremes and the mean with however many absurd phrases, and place the whole essence of any subject vividly before the eyes. (Kepler 1604/2000, p. 109; Kepler 1604/1939, II, p. 92)

When a natural philosopher faces a problem, he commonly assumes that nature is hiding some lawful behavior or harmony. ${ }^{4}$ Kepler recommended facing such a prob-

\footnotetext{
2 From this point onward, this publication will be cited using the name Paralipomena when quoted in the text.

3 See Lohne (1959, p. 117).

4 Cardona (2016a) is a study of the Neoplatonic influence of the concept of harmony in Keplerian heuristics.
} 
lem by contrasting it with an analogy to shed light upon the hidden secret. The main research problems that occupied Kepler's agenda could be formulated in this way: Given the fact that nature, when a particular aspect is considered, behaves lawfully (limited appearance) when it may well behave in any other way (unlimited potentialities), there must be a metaphysical principle in nature that underlies this restriction. Kepler tried to find one or several principles that could restrict these infinite logical possibilities to a few actual (de facto) possibilities. Finding an adequate analogy, from Kepler's perspective, meant finding a mathematical resource, a geometrical diagram for example, that could provide finite control over the infinite and that, despite any differences, would allow the emergence of the same behavior that is manifested in the particular aspect of nature that is under study.

In that order of ideas, analogies come with diagrams that can play the role of tools that lead or control the philosopher's reasonings. One may consider these diagrams as "paper tools" as Arianna Borelli (2017, pp. 60-61) suggests, "[t]hinking with objects [paper tools] means tentatively conceiving the behaviour of as yet unexplored artefacts by conceptualizing them in terms of already known ones". Diagrams, adjusted to the rules of control that the analogy demands, allow us to predict features of the phenomenon we are studying. Borelli adds: "The diagrams fulfilled this function on the one side by defining the structural features shared by a potentially infinite number of individual cases, and the other by demonstrating the empirical adequacy of the results achieved by assuming the validity of those common features" (2017, p. 61). Valeria Giardino refers to these diagrams as cognitive tools, which gather "all cases of two-dimensional representations where their two dimensionality is relevant for the way in which information is displayed and read off from them" (2017, p. 500). Giardino asserts that diagrams used as cognitive tools have more than one role: They are not merely a synoptic picture of the mathematical object in question, but they also suggest some sort of rational intervention. Kepler's use of analogies is a perfect example of the features pointed out by Borelli and Giardino. The application of diagrams in Kepler's heuristics is not reduced to solely illustrating a result, but it is essential to its creation and evaluation. We will show that Kepler's analogies do agree with Giardino and Borelli's analysis. In that sense, diagrams work as tools that are introduced to complex cases (i.e., cases that involve infinite possibilities) to bring to light a metaphysical causality that is initially hidden. Finding an adequate analogy does not necessarily involve inductive work, nor does it deductively follow from any particular metaphysical principles; finding this analogy is a matter of heuristics. In agreement with the complex role of diagrams already identified by Giardino, Heeffer suggests that success in using diagrams demands attention to two aspects: "While diagrams obviously resort to our visual capabilities, they are not limited to mere visual actions but also imply kinetic mental operations. [...] [In addition], diagrams have to be interpreted, as they can be overdetermined or underdetermined for their purpose" (2017, pp. 145-146). We will highlight these two characteristics in Kepler's work.

In the same way that certain quantitative laws for the proportions of the lengths of strings limit the infinite numbers of possible musical sounds to a few harmonic combinations, we can expect the existence of certain mathematical controls that restrict the physical possibilities in order to find the basis for this harmony. Kepler preferred geometrical analogies to arithmetical analogies. Indeed, Walker wrote the following with 
regard to Keplerian methodology: "Analogies based purely on numbers correspond to no archetype in the soul of man or mind of God, whereas geometric analogies do so correspond, and, in many cases, are therefore more than analogies: they display the reasons why God created things as they are and not otherwise" (1978, p. 44). Analogies, when seen as described above, allow God's plan to be seen by the eyes of human reason. ${ }^{5}$

The analogy must be compatible with the empirical information that the philosopher has at hand. However, Kepler was not exactly an investigator that was dedicated to gathering of empirical information. Just as he used empirical data from another expert (Tycho Brahe) for his astronomical investigations, Kepler decided to use the data from Witelo's treatise in his studies on refraction. ${ }^{6}$ In chapter IV of the Paralipomena, Kepler copied a table containing the information that Witelo had obtained from experiments that he had performed when studying the passing of light from air to water. Witelo's table presents the angle of incidence (measured from the normal), the deviation of the refracted ray with respect to the incident ray, and the angle of refraction with respect to the normal. Lohne stated that not only did Witelo plagiarize the work of Alhacen paragraph by paragraph, but also that he took the table from Ptolemy (Table 1). Some of Ptolemy's manuscripts (discovered in 1800) suggest, according to Lohne, that Witelo had slightly modified the empirical information that was collected by Ptolemy. ${ }^{7}$ Lohne also noted that two years after publishing the Paralipomena, thanks to the works of Harriot (1968, pp. 414-426), Kepler found out that certain aspects of Witelo's data were doubtful. $^{8}$

Kepler searched for a diagram or analogy that could enable the prediction of the angle of refraction when one knows the conditions of incidence and also the proportion of the optical densities of the two mediums. He proposed three families of analogies; however, all three failed. With regard to the first family, Kepler concentrated on the cause of the refraction and assumed that the refraction was produced by the difference between the optical densities of the two media. Kepler drew a pair of segments with lengths that had the same proportions as the optical densities of the two media. He then tested several configurations and could not manage to make any of these configurations agree with Witelo's empirical information. With regard to the second family of analogies, Kepler decided to focus on the effects of refraction, rather than on its causes. In this case, he decided to study the formation of images caused by the effects

\footnotetext{
5 Zaiser evaluated Kepler's heuristics in similar terms: "Harmony is present when a multitude of phenomena is regulated by the unity of a mathematical law which expresses a cosmic idea" (1932, p. 47).

6 Frederic Risner (1533-1580) published an influential edition in 1572 called the Opticae thesaurus, with the works of Alhacen and Witelo. The edition serves as an expression of interest in the study of perspective during that time. This work favored the spreading and discussion of the main ideas of both the Arab and British traditions.

7 See Lohne (1959), p. 114.

8 Witelo took the data from Ptolemy, except for a slight difference on the first line (Heeffer 2014, p. 66). Itard holds that Kepler corrected the deviation that was written on the first line so that it would be coherent with the angle of refraction (1957, p. 60). Edward Grant notes that the printed editions of Witelo's Perspectiva have and angle of $7^{\circ} 45^{\prime}$ in the first line; however, he also went through three previous manuscripts in which the angle of the first line is $7^{\circ} 55^{\prime}$ (1974, p. 425, note b of Table 1). Kepler should have corrected the angle of refraction and not the angle of deviation. We will restrict our analysis to Witelo's data (Risner's edition) with Kepler's changes and will assume that it is reliable empirical information.
} 
Table 1 Witelo's data

\begin{tabular}{lll}
\hline Angle of incidence & Deviation & Angle of refraction \\
\hline $10^{\circ}$ & $2^{\circ} 5^{\prime}$ & $7^{\circ} 45^{\prime}$ \\
$20^{\circ}$ & $4^{\circ} 30^{\prime}$ & $15^{\circ} 30^{\prime}$ \\
$30^{\circ}$ & $7^{\circ} 30^{\prime}$ & $22^{\circ} 30^{\prime}$ \\
$40^{\circ}$ & $11^{\circ}$ & $29^{\circ}$ \\
$50^{\circ}$ & $15^{\circ}$ & $35^{\circ}$ \\
$60^{\circ}$ & $19^{\circ} 30^{\prime}$ & $40^{\circ} 30^{\prime}$ \\
$70^{\circ}$ & $24^{\circ} 30^{\prime}$ & $45^{\circ} 30^{\prime}$ \\
$80^{\circ}$ & $30^{\circ}$ & $50^{\circ}$ \\
\hline
\end{tabular}

of refraction. Kepler believed that it would be possible to conceive a conical mirror (hyperbolic, elliptic or parabolic) that would produce, by reflection, the same images that were produced by refraction. The three trials Kepler performed using this analogy failed systematically. With regard to the third family of analogies, Kepler concentrated once again on the causes of refraction. He speculated on the existence of two linked causes, i.e., the difference between the optical densities of the two mediums on the one hand and the resistance produced by the second medium on the other hand. This resistance had to increase as the angle of incidence increased. This third family of analogies led Kepler to defend a complex relationship that agreed with both Witelo's data and the predictions of the Snell-Descartes law, but only at small angles of incidence. ${ }^{9}$ Kepler's law is given by $i-r=k i \sec r$, where $k$ is a proportionality constant, $i$ is the angle of incidence and $r$ is the angle of refraction. ${ }^{10}$ Kepler used this finding to anticipate some of the approximations that allowed him to formulate the theorem that states that it is only possible to accomplish a clear and distinct contemplation of a light source when light coming from a point gathers once again to a point on the retina $\left(1604 / 2000\right.$, p. $182 ; 1604 / 1939$, II, 171) ${ }^{11}$ and to propose some of the axioms used in the interpretation of images formed using telescopes. ${ }^{12}$ Given that this complex law led Kepler to valuable results, this could probably explain why he stopped searching for a simpler law.

In this article, we propose a diagram or "paper tool" that avoids empirical obstructions and that also agrees with the recommendations of the first two families of analogies. We will show that this tool could have anticipated the Snell-Descartes law. As Heeffer suggests, "a geometrical diagram of the measurement of refraction,

\footnotetext{
9 For a detailed study of the strategy of the third family of analogies, see Cardona (2016b).

10 Buchdahl (1972), using a Taylor expansion, showed that Kepler's law could be rewritten using $k=\frac{k^{\prime}-1}{k^{\prime}}$ as:$$
\sin i=k^{\prime} \sin r\left[1-\frac{\left(k^{\prime}-1\right)\left(k^{\prime}-2\right)}{6}(\sin r)^{2}+\cdots\right]
$$

11 "I say that this picture [retinal picture] consists of as many pairs of cones as there are points in the object seen, the pairs always being on the same base, the breadth of the crystalline humor [...] so that one of the cones is set up with its vertex at the point seen and its base at the crystalline [...], the other, with base at the crystalline, common with the former, the vertex at some point of the picture, reaches to the surface of the retina, this too undergoing refraction in departing from the crystalline" (Kepler 1604/2000, 182, 1604/1939, II, 170).

12 With regard to these axioms, see Kepler (1611/2008, axioms VI-VIII, pp. 449-450).
} 
when properly accounting for the exact and co-exact attributes, ${ }^{13}$ becomes itself an instrument that can lead to the discovery of the sine law of refraction" (2017, p. 148). However, while Heeffer focuses on the first family of analogies, we will focus on the two families mentioned. We will also argue that this tool would have agreed with Kepler's metaphysical presuppositions with regard to the nature of light. With this in mind, the paper has the following structure: In the first two sections, we present the first two families of analogies and their empirical obstacles; in the third part, we present our proposal and demonstrate that this new diagram or tool leads to analogies that can suitably adjust both families and that can also anticipate the Snell-Descartes law; in the fourth section, we present Kepler's metaphysical basis that could have accompanied the defense of this tool.

\section{The first family of analogies and its empirical obstacles}

Kepler introduced the first example of the first family in the following terms:

I devised another procedure for measuring, to combine both the density of the medium and the angle of incidence. For since the denser medium becomes the cause of refractions, it therefore seems to be exactly as if one were to extend the depth of that medium, in which the rays are refracted, to a size that the same amount of matter, in the form of the rarer medium, occupies. (Kepler, 1604/2000, p. 101; Kepler, 1604/1939, II, p. 85)

We will now explain Kepler's idea and the accompanying diagram (shown here as Fig. 1). ${ }^{14} A$ represents a source of light, $B C$ is the plane surface that separates air from water (where the second medium is supposed to be optically denser than the first, and thus $\left.n_{2}>n_{1}\right),{ }^{15}$ and $C, F, G$ and $B$ represent the possible points of incidence. Kepler indicates that "[b]ut because it is denser imagine accordingly that the bottom $D E$ is pushed down far enough that there is the same amount of matter in the depth $C K$ under the form of the rarer medium as there is in the depth $C E$ under the form of the denser" $(1604 / 2000$, p. 101; 1604/1939, II, 85). According to our reading of this passage, Kepler asked readers to imagine an arbitrary depth of water, $C E$, and suggested identification of a new depth $C K$ in such a way that the segments $C E$ and $C K$ have the same ratio as the optical densities of the two media: $\frac{C K}{C E}=\frac{n_{2}}{n_{1}}=n_{21}$. Kepler then imagined that the water contained at the depth $C E$ then extended homogeneously until it filled the new depth $C K$. Under these conditions, the distribution of the water's mass would accommodate the air's density. Therefore, there would be no difference between the densities of the two media, and the light from $A$ would thus follow the direct paths $A D, A I$ and $A H$. This behavior occurs provided that the optical density

\footnotetext{
13 Heeffer takes the distinction between exact and co-exact from Manders (2008). "Co-exact attributes are those conditions which are unaffected by some range of every continuous variation of a specified diagram [...]. Exact attributes are those which, for at least some continuous variations of the diagram, obtain only in isolated cases" (Manders, p. 92).

14 Heeffer has pointed out that Kepler's figure has a family resemblance with a figure suggested by Della Porta in De refractione optices; cfr. (2017, pp. 162-163).

15 We will use the common and modern expression $n_{21}$ to refer to the quotient of the two optical densities (refractive indexes) $\frac{n_{2}}{n_{1}}$.
} 


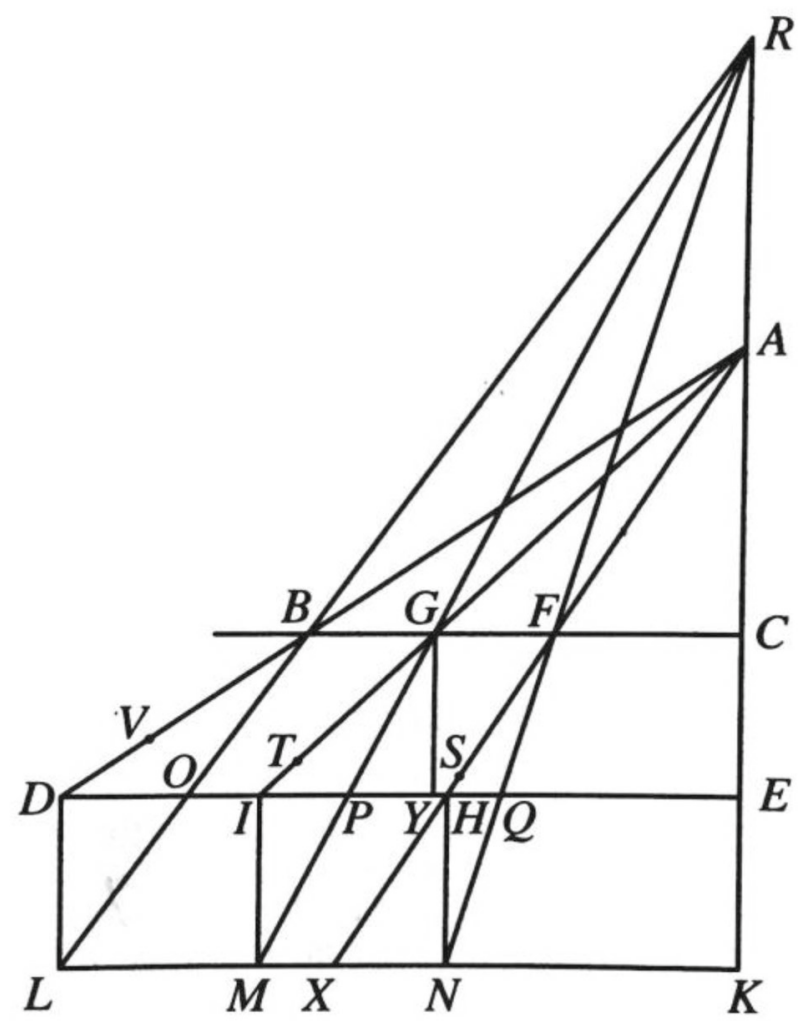

Fig. 1 First family of analogies (Kepler 1604/2000, 101, 1604/1939, II, 86)

remains proportional to the material density. ${ }^{16}$ Kepler then drew perpendicular lines to $D E$ from $D, I, H$ and $E$ to obtain their intersections with $L K$, i.e., $L, M, N$ and $K$. Next, Kepler drew the segments $L B, M G$ and $N F$. Finally, he assumed that the diagram would allow him to predict the path of the light. Therefore, if the light traveled from air to water, it would follow similar paths to $A B L, A G M, A F N$ and $A C K$; if it traveled from water to air, it would follow the inverse paths. The diagram can be used to explain why, when $n_{2}>n_{1}$, the refracted ray comes closer to the normal, and also why, when $n_{2}<n_{1}$, the refracted ray comes closer to the surface. Indeed, if $C K>C E$, then the points $L, M$ and $N$ are located beneath $D E$; otherwise they are located above that line.

If $L, M, N$ and $K$ are objects lying at the depth $L K$, then according to the classical principle for the formation of images, ${ }^{17}$ the images that are seen by and observer at $A$ must be located at $D, T, S$ and $E$, respectively. Following that concept, all objects lying

\footnotetext{
16 Harriot collected valuable empirical information to show that there are no reasons to expect the refraction to be dependent on differences in the material densities. We will comment on these experiments later.

17 This principle, which was formulated by Euclid and Ptolemy, states that the image of an object is formed at the point of intersection of the reflected (or refracted) ray and the normal that passes through the object, known as the cathetus. Kepler formulated a severe criticism of this principle (Kepler 1604/2000, III,
} 


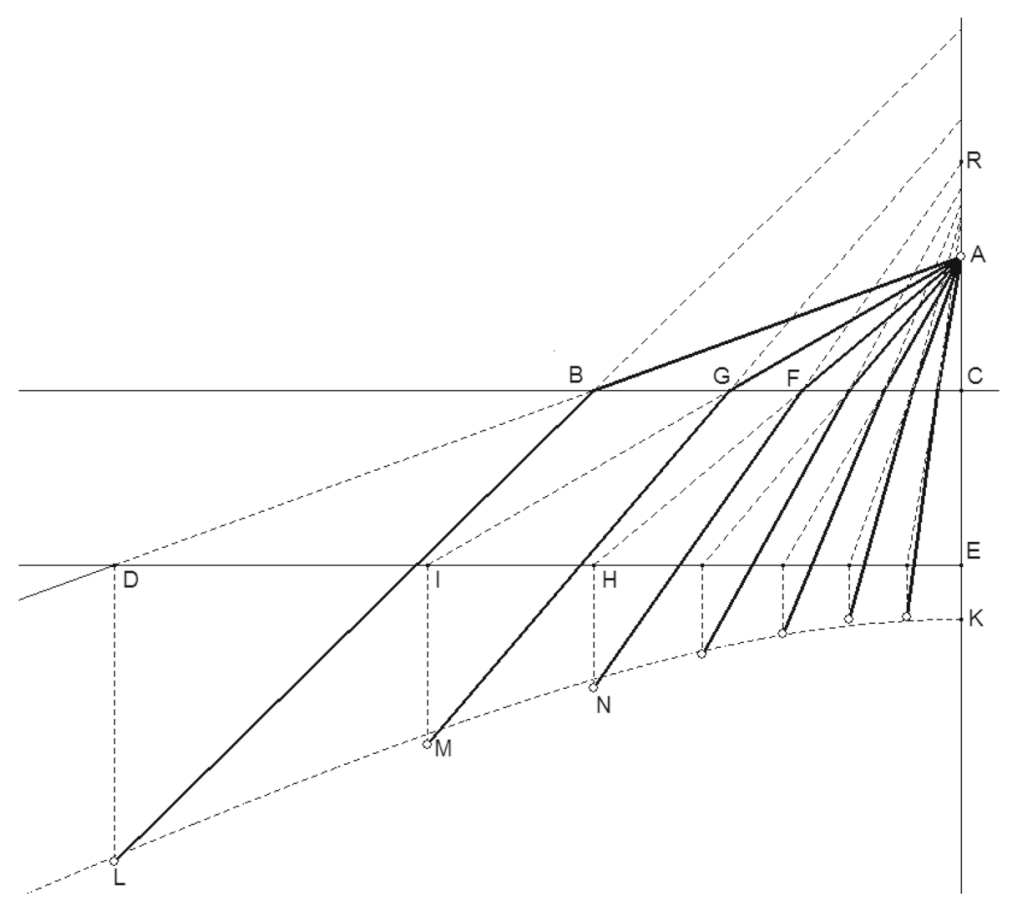

Fig. 2 Evaluation of the first analogy

at the depth $L K$ must be perceived at the same depth of $D E$, regardless of the angle of incidence. The rule that is implicit in Kepler's proposal takes the form $\frac{\tan i}{\tan r}=n_{21}$. From this, it can be inferred that, if we consider the point of incidence $F$, for example, we find that the point $R$, which is obtained from the intersection of the extension of $F N$ and the normal $C K$, is independent of the specific location of $F$. Therefore, if this analogy is correct, one can expect that every extension of the type of $N F, M G$ and $L B$ will meet at $R$ (Fig. 1) (Cfr. Kepler 1604/2000, IV, pp. 100-102; Kepler 1604/1939, pp. 84-86).

"This way of measuring," says Kepler about the analogy, "is refuted by experience" (1604/2000, p. 102, 1604/1939, II, 86). Figure 2 is constructed using the empirical information that was gathered by Witelo; we will use this figure to show the type of refutation that Kepler was discussing. The segment $C E$ represents an arbitrary depth, while $E D$ is perpendicular to $C E$. $F$ is any point of incidence that can be taken from Witelo's values. $A F$ represents an incident ray, $N F$ the refracted ray according to Witelo's data. $N$ is the intersection of the refracted ray and the perpendicular to $D E$ through $H$, which is the intersection between $E D$ and the extension of $A F$. Therefore, $N$ is the place of the image of an object $H$ seen by an observer located at $A$. If we repeat the same procedure for each pair of data, we will obtain the following results which contradict the theoretical expectations deduced from the first exemplar of the

Footnote 17 continued

prop. 17; Kepler 1604/1939, pp. 85-86, 68-69). However, for the purposes of this article, these objections can be omitted. 
first family of analogies: (1) The images $N$ of the objects $H$ which are at the same depth $C E$ under water are not observed at the same depth; the location of the images seen from the air varies depending on the angle of incidence; (2) the extensions $N F$ do not meet at one point on the normal $A C$ : "And if you examine the angles of refraction from Witelo and Tycho, the directing point $R$ of the refracted rays $B O, G P, F Q$, is not one" (1604/2000, p. 102, 1604/1939, II, 86). Figure 2 indicates the approximate geometrical placement of the points $N$ when $F$ is varied. This place is very different from the straight line demanded by the analogy (i.e., $K L$ in Fig. 1). However, when the angles of incidence are very small, the geometrical placement seems to behave as suggested by the analogy. It is important to keep in mind that the geometrical locus of $N$ looks like a hyperbola. Later we will show that it is indeed a hyperbola. When Kepler was gathering arguments in order to explore the possibility of hyperbolas, he stated the following suggestion: "For if you designate with points the places of the images in water through all the angles of inclination, a hyperbola will be approximately foreshadowed, which increases my confidence" (1604/2000, p. 111, 1604/1939, II, 97).This aspect will be fundamental to the work ahead.

Heeffer believes that Kepler would have arrived at the law of the sines if he had proceeded in the following way $\left(2017\right.$, pp. $158-159 ; 2014$, p. 71) ${ }^{18}$ First, to demand that just as all incident rays $(A F, A G, A B)$ meet in $A$, all the extensions of the refracted rays $(N F, M G, L B)$ meet in a point $R$ (Fig. 1). Second, for each point of incidence $F$, $G, B$, to locate the corresponding points $H, I, D$ in the intersection of the extension of the incident rays with the parallel to $B C$, plotted at an arbitrary depth $C E$. Finally, to chose the points $N, M$ and $L$, respectively, on the lines $R F, R G$ and $R B$ in a way that $\frac{F R}{F A}=\frac{F N}{F H}, \frac{G R}{G A}=\frac{G M}{G I}$ y $\frac{B R}{B A}=\frac{B L}{B D}$. Heeffer trusts that each of these ratios coincides with the ratio of the optical densities. ${ }^{19}$ However, if we assume that $R$ is invariant, the points $N, M, L$ are spread on a perpendicular to $A C$, and the quotient that remains invariant is $\frac{\tan i}{\tan r}$ and not $\frac{\sin i}{\sin r}$ as Heffer claims. Figure 2, constructed with Witelo's data, shows that the extensions of the refracted rays do not meet in a point. Heeffer's proposal, taking an arbitrary depth $C E$, would imply the correct invariance (the law of the sines) if one insists that $\frac{F R}{F A}=\frac{F N}{F H}$ for each $F$, without demanding that $R$ must be the same for each point of incidence. Therefore, Heeffer makes a mistake in identifying the co-exact attribute that would lead to the law of the sines.

The first family of analogies obeys the following scheme: Draw a pair of segments in a geometrical arrangement in a manner such that (1) their lengths stand with the same proportion as the optical densities of the two media, and (2) the diagram (paper tool) anticipates the course that a ray of light coming from $A$ would follow when passing from one medium to another. Kepler then suggested twelve examples that were adjusted with respect to the scheme described. Heeffer summarized 11 of the 12 examples in the following table. We added the hypothesis $H_{0}$ which is not on the list and which corresponds precisely to the conjecture that we have carefully examined ${ }^{20}$ :

\footnotetext{
18 Our presentation, though it shares the same spirit, slightly differs from Heeffer's.

19 "This ratio is a co-exact property of the geometrical diagram and remains invariant with respect to the angles of incidence. So is the ratio of the sines of angles $G A C$ and $G R C$ or $G R / G A$ also equal to $F R / F A$. This unfortunate oversight was Kepler's failure in discovering the sine law" (Heeffer 2017, p. 159).

20 The names of the geometrical elements can be taken from Fig. 1 (cfr. Heeffer 2014, p. 70, tabla 4.7). The hypothesis are systematically stated in Kepler (1604/2000, pp. 102-104, 1604/1939, II, 87-89).
} 


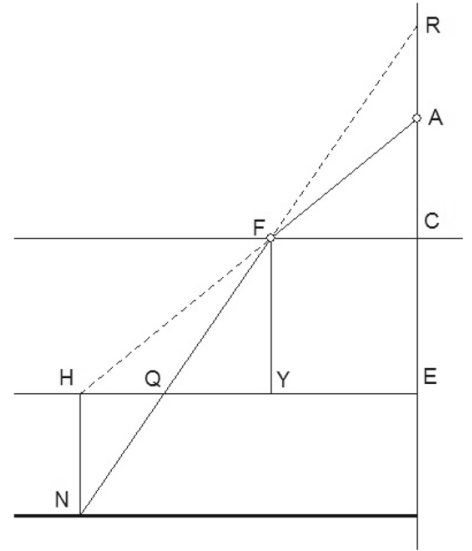

(a)

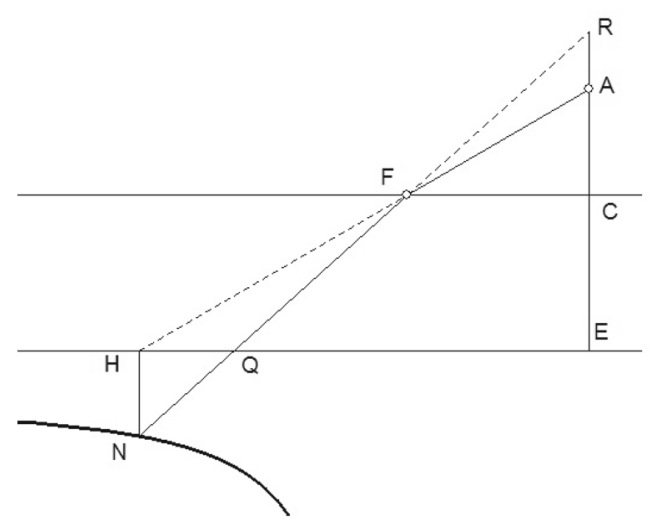

(b)

Fig. 3 Candidates for the first family of analogies

Table 2 Conjectures of the first family of analogies

\begin{tabular}{ll}
\hline Hypothesis & $\begin{array}{l}\text { Quotient that represents the ratio } \\
\text { between the optical densities }\end{array}$ \\
\hline$H_{0}$ & $\mathrm{CK} / \mathrm{CE}$ \\
$H_{1}$ & $\mathrm{EQ} / \mathrm{EH}$ \\
$H_{2}$ & $\mathrm{FQ} / \mathrm{FH}$ \\
$H_{3}$ & $(\mathrm{EQ})^{2} /(\mathrm{EH})^{2}$ \\
$H_{4}$ & $\mathrm{a} \mathrm{EQ} / \mathrm{b} \mathrm{EH}$ \\
$H_{5}$ & $(\mathrm{FHEC}) /(\mathrm{FQEC})$ \\
$H_{6}$ & $(\mathrm{EQ})^{3} /(\mathrm{EH})^{3}$ \\
$H_{7}$ & $\mathrm{IY} / \mathrm{IP}$ \\
$H_{8}$ & $\mathrm{GC} / \mathrm{IE}$ \\
$H_{9}$ & $\mathrm{CE} / \mathrm{CK}$ \\
$H_{10}$ & $\mathrm{FH} / \mathrm{FX}$ \\
$H_{11}$ & $\mathrm{CK} / \mathrm{FX}$ \\
\hline
\end{tabular}

It is easy to see that $H_{0}$ and $H_{1}$ are equivalent, and both lead to the invariance $\frac{\tan i}{\tan r}=n_{21}$. Although $H_{o}$ is similar to $H_{9}$, in the latter Kepler located the points $S, T$ and $V$ on the extensions $A F, A G$ and $A B$, respectively, in such a way that $C E=F S=$ $G T=B V$. These points are suggested as the place of the images of the objects lying on $K L$. Figure $3 \mathrm{a}$ and $\mathrm{b}$ shows the results of the evaluation of $H_{1}$ and $H_{2} \cdot{ }^{21}$ In addition, Kepler wondered if the images could rise in proportion to the sines of incidence. He rapidly dismissed this option because, in that case, the location of the images would be identical in every medium. This then led him to suggest that the rise of the location of the images could depend on two factors: first, a perpendicular elevation in proportion to the quotient of the optical densities of the media and, second, an additional rise

$21 H_{2}$ equivalent to $\frac{\sec i}{\sec r}=n_{21}$. 
proportional to the angles of incidence (1604/2000, p. 104, 1604/1939, II, 89). A combination of this style was rehearsed in the third family of analogies. ${ }^{22}$

Each of the prototypes for the first family led to similar conflicts with the empirical evidence. With regard to this aspect, Kepler concluded that: "Hitherto, we have followed an almost blind plan of enquiry, and have called upon luck. From now on let us open the other eye, proceeding with a sure method" (Kepler 1604/2000, p. 104, $1604 / 1939$, II, 88). For the empirical trials, Kepler kept in mind the locations of the images of a known object or the location that an object must have to allow its image to be perceived at equal depths at all times. These difficulties led Kepler to revert the order of his reasoning. In other words, Kepler took the locations of the images that were produced by refraction but used them as starting points to suggest new analogies rather than as a key for the empirical evaluation.

\section{The second family of analogies and its obstacles}

Because images on mirrors are also formed in places that differ from the real locations of the objects, Kepler believed that mirrors, for which the laws of reflection were well known, could provide the tool or model that he required. Kepler summarized this new course of investigation in the following terms: "And indeed, this very difficult Gordian Knot of catoptrics I finally cut by analogy alone. [...] when I consider what would happen in mirrors, and what fittingly should happen in water following this similitude" (1604/2000, p. 105, 1604/1939, II, p. 88). Kepler's fundamental idea consisted of imagining that a plane surface that separates two media with different optical densities could be conceived as a reflective conic surface that could lead to a rule of control that would restrict the infinite possibilities of refraction. The argument that persuaded Kepler to think of conic mirrors was the following:

In the same manner that the image of an object is made smaller in convex mirror, it is also thus in rarer media; and as the image is made greater in concave mirrors, it is also thus in denser media. In convex mirrors, the middle parts of the image get closer than the surrounding parts, while in concave mirrors they get farther away. The same thing occurs in different media, so that in water the bottom seems lowered, the surrounding parts raised. Hence it was apparent that a denser medium corresponds to a concave mirror surface, and a rarer medium to a convex surface. It was therewith evident that the plane surface of water assumes a certain type of curvature. (1604/2000, p. 105; 1604/1939, II, 91)

Next, we will focus on the main structure of Kepler's new strategy. Let us imagine that $B$ and $A$ are the two foci of a hyperbola and that $O$ is both the origin of the coordinate system and the midpoint between $A$ and $B$ (Fig. 4). Kepler took into consideration the pair of data of incidence and refraction $\left(80^{\circ}, 50^{\circ}\right)$ from Witelo's table and proceeded to calibrate the hyperbolic curve of the new model. He used the points $A$ and $B$ to construct a triangle that modeled the chosen pair of data. He then located the point $C$ such that the angle $A B C$ measured $80^{\circ}$ and the angle $B A C$ measured $50^{\circ}$. If we then

22 Cardona (2016b). 


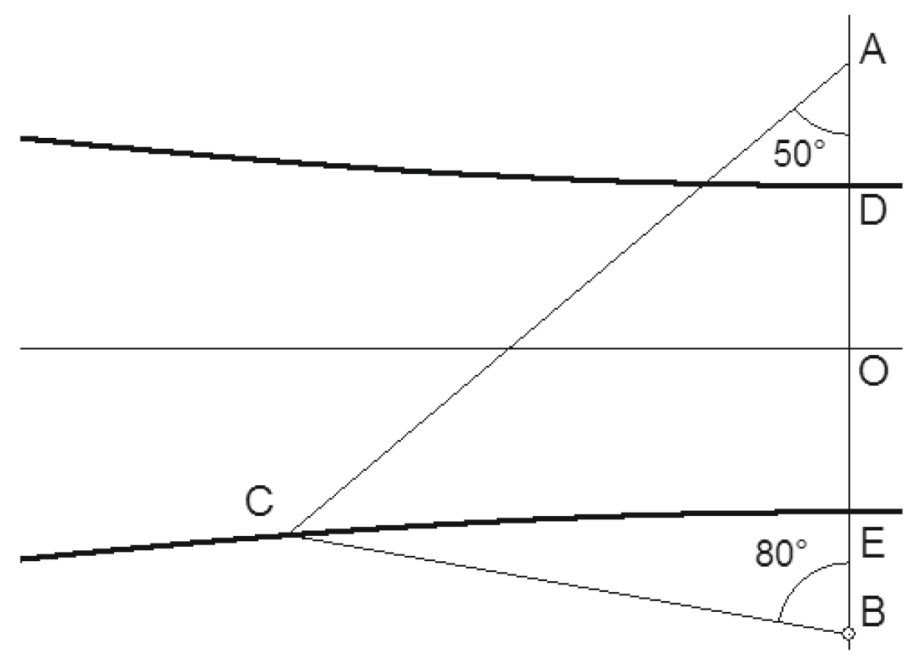

Fig. 4 Modeling using hyperbolas

imagine that $A$ and $B$ are given, it is then possible to build the hyperbola that passes through $C$ and has foci at $A$ and $B$. With the available information, we can calculate the magnitudes of $A C$ and $C B$ and, based on these values, the difference $A C-C B$. If we assume that the length of the parameter $A B$ is $2 f$, then $A C=\frac{\sin 80}{\sin 50} 2 f$. Since the triangle $A B C$ is isosceles, $B C=2 f$. Therefore, $A C-B C=2 f\left[\left(\frac{\sin 80}{\sin 50}\right)-1\right]$. Kepler knows that at each point $C$ of a hyperbola, the difference in the lengths $A C$ and $B C$ is the same regardless of the choice of $C$. Thus, the difference $A C-C B$ determines the length $D E$, with $D$ and $E$ as vertices of the hyperbola.

Next, Kepler examined whether the diagram would allow the prediction of the angle of refraction for any of the angles of incidence contained among Witelo's data. Let us take an arbitrary point $F$ on the hyperbola (Fig. 5); then, let us draw the lines $F B$ and $F A$. Next, we measure the angles $A B F(\alpha)$ and $F A B(\beta)$ with the hope that these angles will behave in agreement with the expectations imposed by Witelo's information. ${ }^{23}$

For Kepler, the results of this approach could not have been more discouraging. In Fig. 6, we have drawn, for any arbitrary angle $A B F$, the angle $B A F^{\prime}$ that would be expected to agree with the Snell-Descartes law for a refractive index that has been adjusted with respect to the pair of data $\left(80^{\circ}, 50^{\circ}\right)$. In the figure, $F^{\prime}$ is the point of intersection of the lines $B F$ and $A F^{\prime}$. The dashed lines show the geometrical locus of the points of intersection when the angles of incidence are varied from $0^{\circ}$ to $90^{\circ}$. The small crosses indicate the intersection values when adjusted with respect to the empirical information collected by Witelo. These data are distributed very closely to the geometrical locus and are located notably far away from Kepler's hyperbola. Kepler therefore had to abandon this method of conceiving the required diagram.

Subsequently, Kepler tried to conceive a new diagram using an ellipse: "You could now guess immediately," Kepler insisted, "that because the hyperbola does the opposite

\footnotetext{
23 It can be proved without difficulty that the behavior of these angles is independent of the choice of the parameter $A B$.
} 


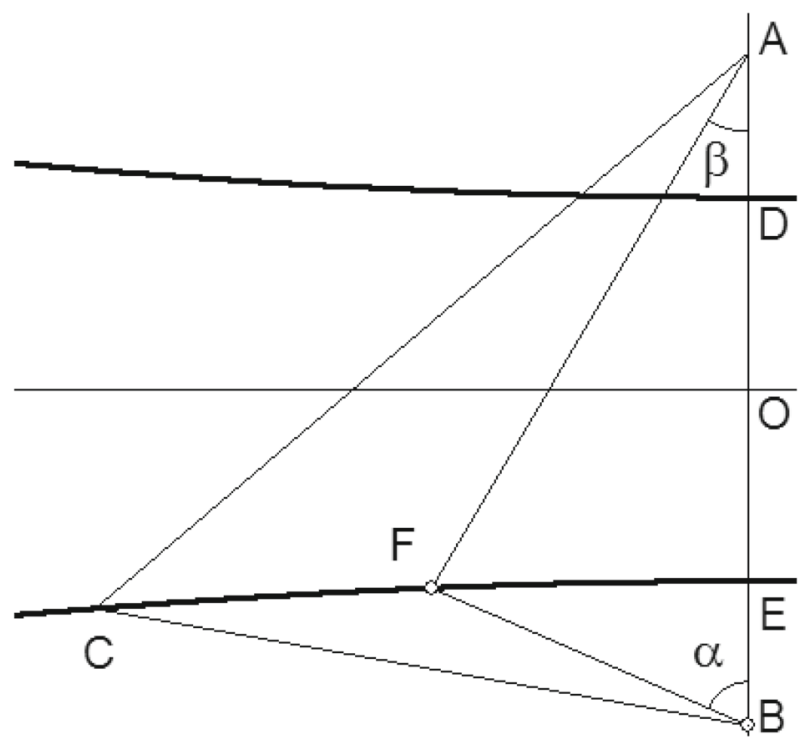

Fig. 5 Trial with arbitrary point $F$

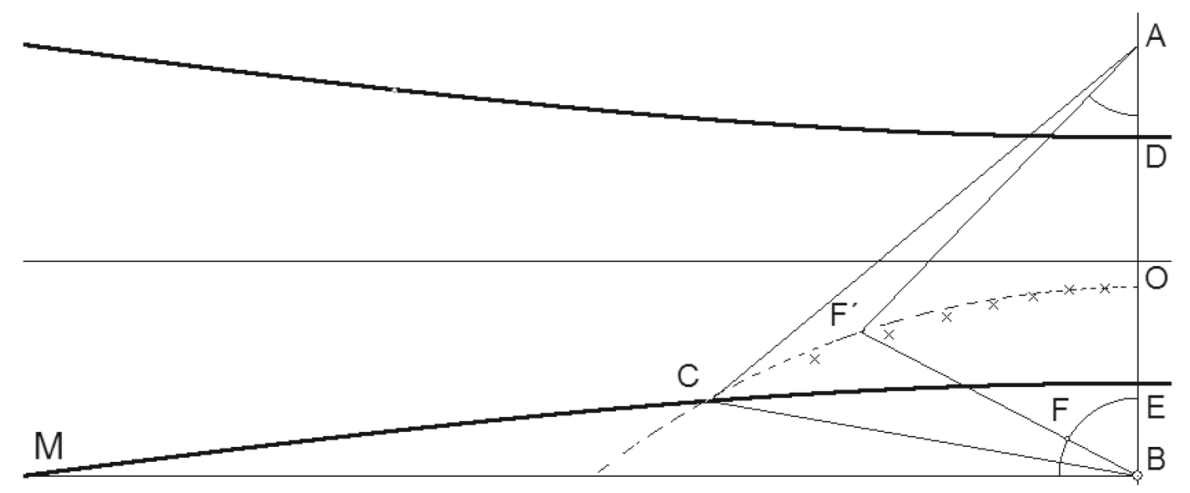

Fig. 6 Comparison between the results of the approaches of Kepler (black lines), Witelo (crosses), and the Snell-Descartes law (dashed line)

of the refractions, the ellipse, being the hyperbola's opposite, is going to do the same as the refractions, and will accommodate itself to the measure" (1604/2000, p. 112, $1604 / 1939$, II, p. 95). Now, let us imagine that $B$ and $A$ are the two foci of an ellipse, while $O$ is both the center of the coordinate system and the midpoint between $A$ and $B$ (Fig. 7). Kepler repeated the previous procedure, except for the fact that he used an ellipse in place of the hyperbola. The data that were adjusted to the Snell-Descartes law are drawn using dotted lines, and the intersection values expected based on the information from Witelo are represented by crosses. These data move away from the expected ellipse. 


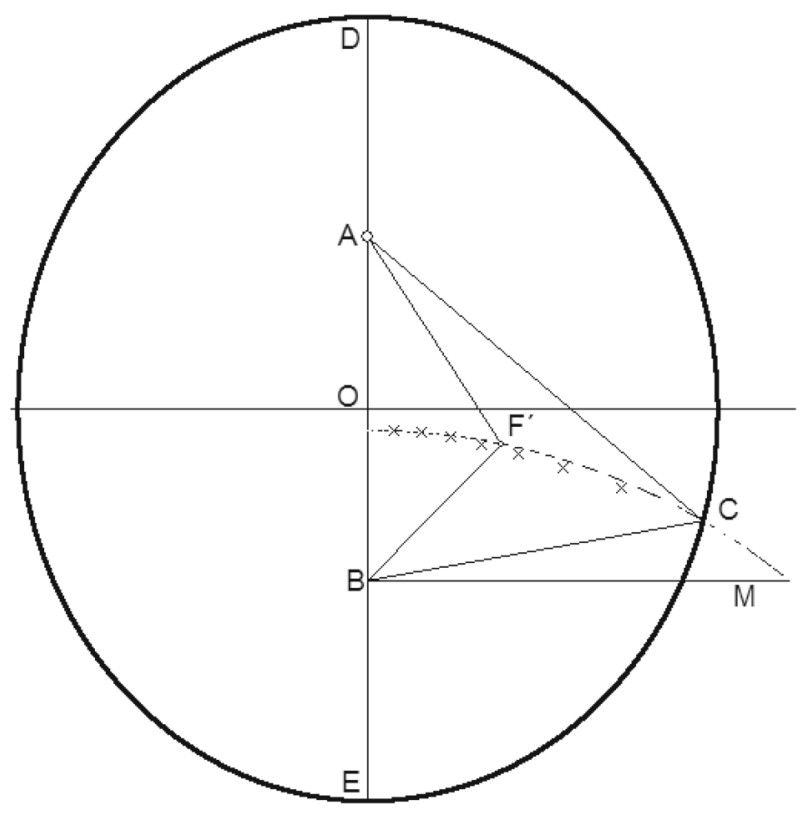

Fig. 7 Comparison between the results of the approaches of Kepler (black line), Witelo (crosses), and the Snell-Descartes law (dotted line)

Kepler then decided to try using a parabola. Given that he was unable to deal with two foci, he suggested a few modifications to the manner in which the paper tool or model was to be conceived. Sadly, the results were again unsatisfactory. However, Kepler left open the possibility of constructing a hyperbola that agreed with the empirical evidence in a similar manner in which he built the parabola. We will return to this matter in the next section.

\section{A defense of a new model}

None of Kepler's trials to find either an appropriate layout of segments or conic surfaces produced encouraging results. Nevertheless, we can still ask how, without abandoning the spirit of his inquiry, Kepler may have proceeded to find a tool that could have led him closer both to Witelo's data and to the law that was later formulated by Descartes and Snell. First, we will present, step by step, the tool that we would like to propose as an alternative. Second, we will search for the type of justification that a Keplerian scientist may have had in mind to conceive the required model. We will then try to show that it is possible to combine a pair of segments with lengths that have the same proportion as the optical densities to suggest a protocol that allows one to the behavior of light. We will then also prove that this model leads to the construction of a hyperbola that opens a way to conceive a second procedure that implies the same result. Both versions of the model can be calibrated using reliable empirical data. When we select 


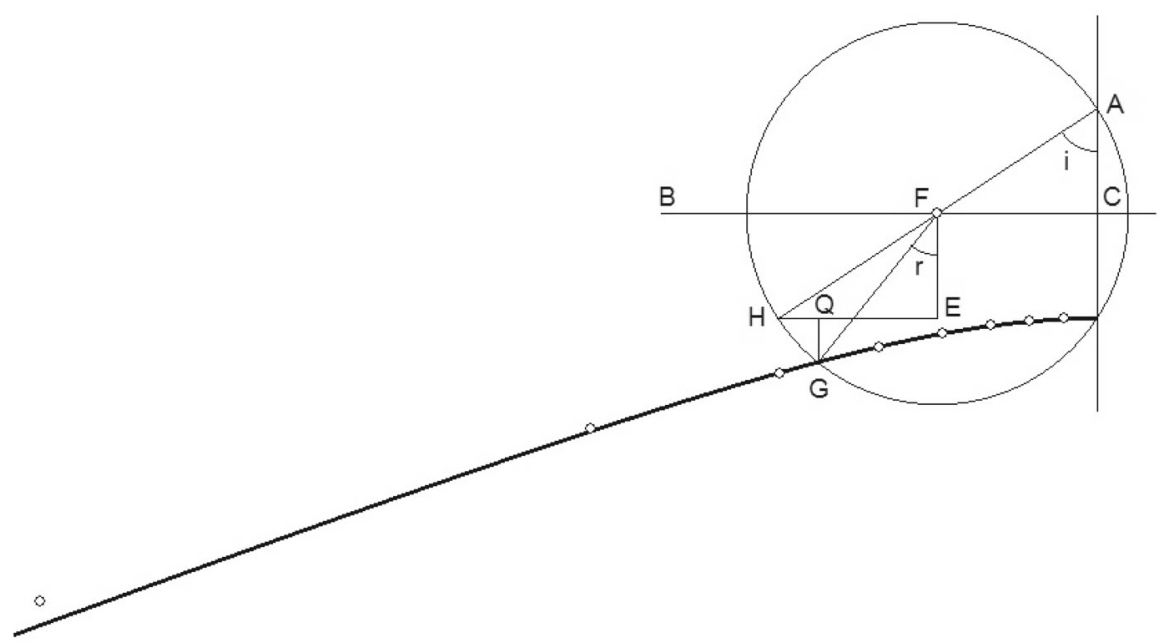

Fig. 8 New diagram or "paper tool" (where the small white circles correspond to Witelo's data)

these data, it will provide information with regard to the quotient of the optical densities and will also allow the remaining empirical information to be predicted.

We believe that Kepler could have followed the path described here. Let $A$ be a light source, $B C$ the surface between two media, and let $A F$ be an incident ray passing from air to water (Fig. 8). Let us then construct a circle centered at $F$ and with radius $F A$. Next, we extend the incident ray until it cuts the circumference at point $H$. The path $F H$ represents the direction of the light propagation if the new medium is homogeneous with the previous medium. We then draw the normal $F E$ and another perpendicular to this line through $H$. Next, on the line $H E$, we locate a point $Q$ such that the quotient $\frac{H E}{O E}$ coincides with the quotient of the optical densities of the two media, i.e., $n_{21}$. Then, we erect the perpendicular line to $H E$ through $Q$ and obtain the point $G$ where this perpendicular intersects the circle. The ray $F G$ represents the path designated for the refracted ray. ${ }^{24}$ The figure shows the geometrical locus of the point $G$ when $F$ moves along the surface separating the two media. Witelo's data are represented by white circles in the figure. The farthest data from the curve that satisfy the Snell-Descartes law are, precisely, the pair that Kepler used to calibrate his model. It is simple to demonstrate that the implicit law used in the construction of this diagram is: $\frac{\sin i}{\sin r}=\frac{n_{2}}{n_{1}}=n_{21}$.

The geometrical locus that is shown in the figure resembles a hyperbola with one of its vertices located at point $A$. We will now show that this is indeed a hyperbola. We assume that $A$ is located on the vertical axis of a coordinate system where the horizontal axis represents the separating surface. Therefore, the vertex $A$ of the conic that we will construct is located at the coordinates $(0, a)$. Let $R$ be the magnitude

24 It is easy to demonstrate that determination of the direction $F G$ is independent of the radius of the circumference. However, for the subsequent analysis, it is convenient to fix the radiant point of light, which in this case is $A$, and simultaneously impose a rule to vary the size of the circle that is being considered. See that we are offering a sophisticated variation of hypothesis $H_{1}$ from Table 2. 
of the radius $F A$. $G$ represents any point on the locus with coordinates $(x, y)$. These coordinates, as stated in the parametric equations, are:

$$
\begin{gathered}
x=a \tan i+\frac{a \tan i}{n_{21}}=a\left(\frac{n_{21}+1}{n_{21}}\right) \tan i \\
y=\sqrt{R^{2}-\left(\frac{a \tan i}{n_{21}}\right)^{2}}=\frac{a}{n_{21} \cos i} \sqrt{n_{21}^{2}-(\sin i)^{2}}
\end{gathered}
$$

It can be proved that, if we assume that $b^{2}=\frac{n_{21}+1}{n_{21}-1} a^{2}$, the geometrical locus of the points $G$ adjusts to the standard equation for a hyperbola, i.e., $\frac{y^{2}}{a^{2}}-\frac{x^{2}}{b^{2}}=1$. This hyperbola has a focal distance $f$ that satisfies the following equation: $f^{2}=a^{2}+b^{2}=$ $\frac{2 n_{21}}{n_{21}-1} a^{2}$. The hyperbola that we have found is the hyperbola that Kepler sought. This hyperbola models the refraction expected when passing from air to water. Indeed, despite the failures detailed above, Kepler never stopped considering how it would have been possible to build a hyperbola for each different medium: "I did not leave it untried to ask whether for any medium there might be its own hyperbola" (Kepler 1604/2000, IV, p. 111, 1604/1939, p. 94).

Kepler calibrated his conics with the pair $\left(80^{\circ}, 50^{\circ}\right)$ because he thought it was the data with the least experimental error (Kepler 1604/2000, IV, prop. 8, p. 127, $1604 / 1939$, p. 108). Figure 8 shows, however, that this pair is located farthest from the hyperbola. ${ }^{25}$ For that reason, we have decided to calibrate the hyperbola using another data pair. We considered Witelo's information and found that the pair of data with the smallest deviation from the expectations of the Snell-Descartes law is $\left(30^{\circ}\right.$, $\left.22^{\circ} 30^{\prime}\right)$. In this case, the estimated refractive index is $1.3065 \pm 0.0024$. Therefore, the tool that adjusts most closely to the second family of analogies and best embraces the Snell-Descartes law can be calibrated using these data. By adopting the procedure from Fig. 8, the expected curve can be built as follows. First, a source of light $A$ is selected on the vertical axis of a coordinate system with origin $O$ (Fig. 9). Point $A$ is located at a distance $a$ from the origin. Then, point $F$ is located on the horizontal axis such that the angle $O A F$ measures $30^{\circ}$. Next, one builds the circumference with center in $F$ and radius $F A$ and traces the perpendicular to the horizontal axe through $F$, that is, $F P$.

Then, we locate point $G$ on the circumference of the circle while ensuring that the angle $P F G$ measures $22.5^{\circ}$. Finally, the hyperbola with its vertex at $A$ that passes through a point $G$ is built. Figure 9 shows this hyperbola, its basic parameters $a$ and $b$, and one of its focal points, $F_{1}$. The hyperbola corresponds to the transition from air to water.

\footnotetext{
25 Harriot had known since 1597 that Witelo had an error of $2^{\circ} 29^{\prime}$ in the measured deviation when the angle of incidence was $50^{\circ}$; cfr. J. Kepler (1604-1611/2008, p. 333). After completing the Paralipomena, Kepler found out about the inaccuracy of Witelo's data. In a letter from 1606, Kepler wrote to Harriot: "I have heard that your experiments differ by two or three degrees from Witelo's data, so I hope to receive the most accurate results, not only with regard to the media, but also with regard to the instruments." (1606-1609/2008, p. 310). (Personal translation).
} 


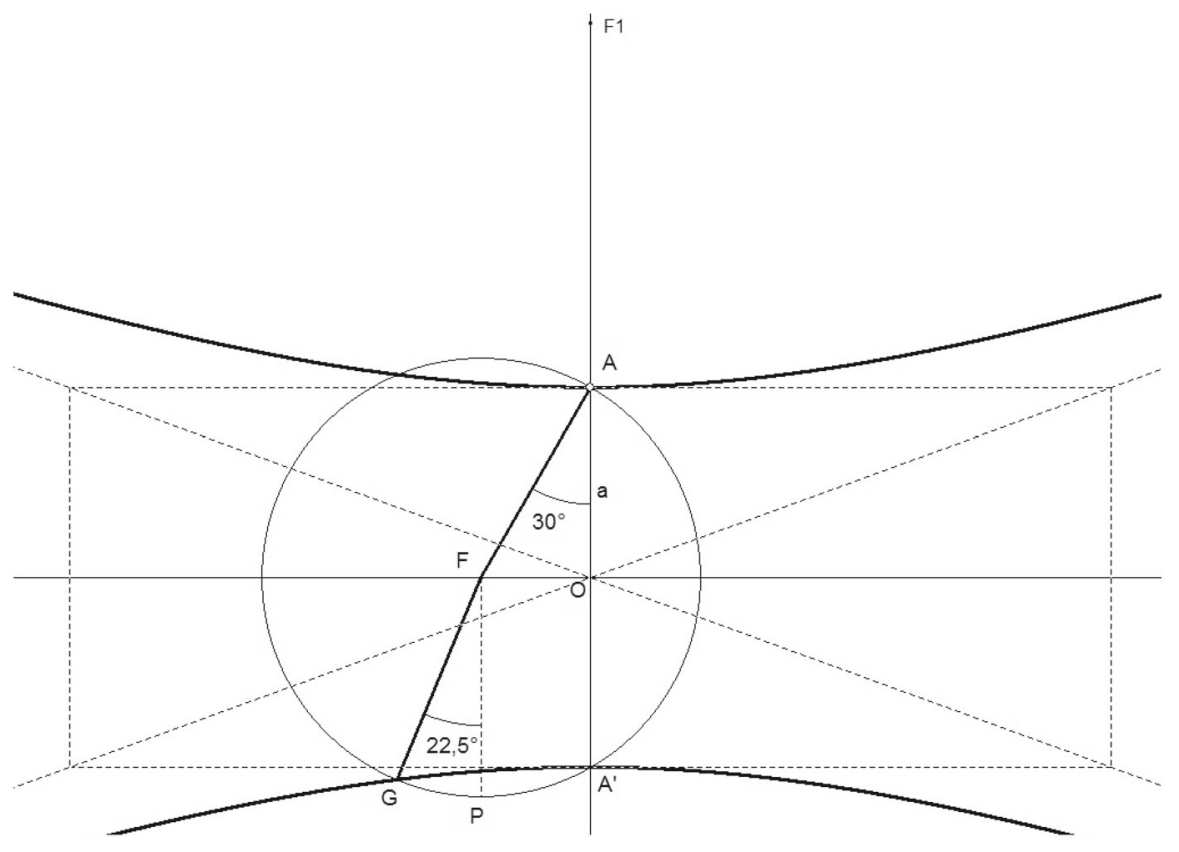

Fig. 9 Calibration of the hyperbola

Given the chosen calibration pair, the parameter $b$ equates to $2.76 a$ and the equation of the hyperbola then becomes ${ }^{26}$ :

$$
\frac{y^{2}}{a^{2}}-\frac{x^{2}}{7.61 a^{2}}=1
$$

The proposed diagram can be used to predict the angle of refraction for any angle of incidence if we perform the following procedure. With the calibrated hyperbola at hand, let us fix any point $C$ on the surface between the two media at which a light ray coming from $A$ cuts the surface (Fig. 10). $E G$ is the normal through $C$. The angle $A C E$ is the angle of incidence. Next, one draws the circle with center $C$ and radius $C A$. Then, one looks for the cut of the circumference with the hyperbola, that is, $D .{ }^{27}$ The angle $G C D$ represents the sought angle of refraction. Figure 10 also shows the information provided by Witelo using white circles.

We can now obtain the geometrical locus of the objects that lie under water and that are viewed from point $A$ as if they were at the same depth $O A^{\prime}$ (Fig. 11). Let $F$ be a point of incidence on the surface. Point $A$ now represents the location of an observer rather than a source of light. The direction of the ray that passes from the water to the air and then passes straight to point $A$ can be anticipated using the procedure illustrated

\footnotetext{
26 The estimation of the angle of refraction is independent of the choice of parameter $a$.

27 Two points of intersection coincide with the vertexes of the hyperbola. The other two points are found symmetrically, with one located on the superior branch and the other located on the inferior branch of the hyperbola.
} 


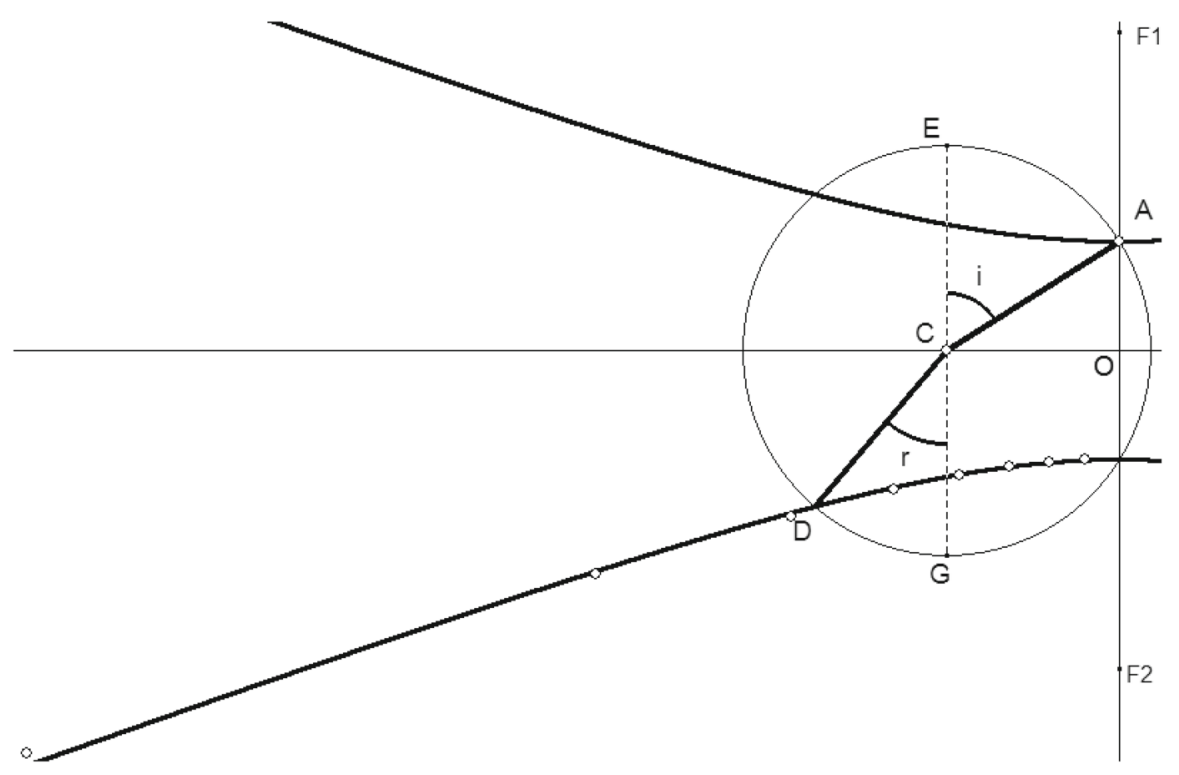

Fig. 10 Use of the proposed model

in Fig. 8. This direction is indicated by $G F$. Next, $G F$ is extended until it cuts the line perpendicular to $H A^{\prime}$ through $H$. This intersection, at point $N$, represents an object whose image is observed from $A$ at the depth $O A^{\prime}$. Figure 11 shows the geometrical locus of any point $N$ when the location of point $F$ varies. By following a very similar procedure to that which was used on the preceding pages, it can be shown that this locus is a hyperbola with parameters $O M=a n_{21}$ and $b^{2}=\frac{4 n_{21}^{2} a^{2}}{n_{21}^{2}-1} ;{ }^{28} M$ is one of the vertexes of the hyperbola. Kepler, as we had already mentioned, anticipated the existence of this hyperbola in the Paralipomena (1604/2000, IV, p. 111, 1604/1939, p. 94). It is likely that Kepler had in mind a geometrical locus similar to the one shown in Fig. 2.

In Fig. $11, R$ is the intersection of the extension of $N F$ with the vertical axe $O A$. One immediately sees that the triangles $H F N$ and $A F R$ are similar; therefore, $\frac{F R}{F A}=\frac{F N}{F H}$. Moreover,

$$
\begin{aligned}
& H E=F S=F H \sin r \\
& Q E=F T=F G \sin i
\end{aligned}
$$

\section{Given that $F H \cong F G$, then ${ }^{29}$}

\footnotetext{
28 The parametric equations for the coordinates of point $N$ are $y=a \tan i \cot r=a n_{21} \frac{\cos r}{\cos i}$ (where $a$ is the parameter that designates the location of point $A$ and the location of the vertex of the hyperbola of calibration that corresponds to the specific medium).

29 Assuming the reversibility of refraction, $n_{12}=\frac{1}{n_{21}}$.
} 


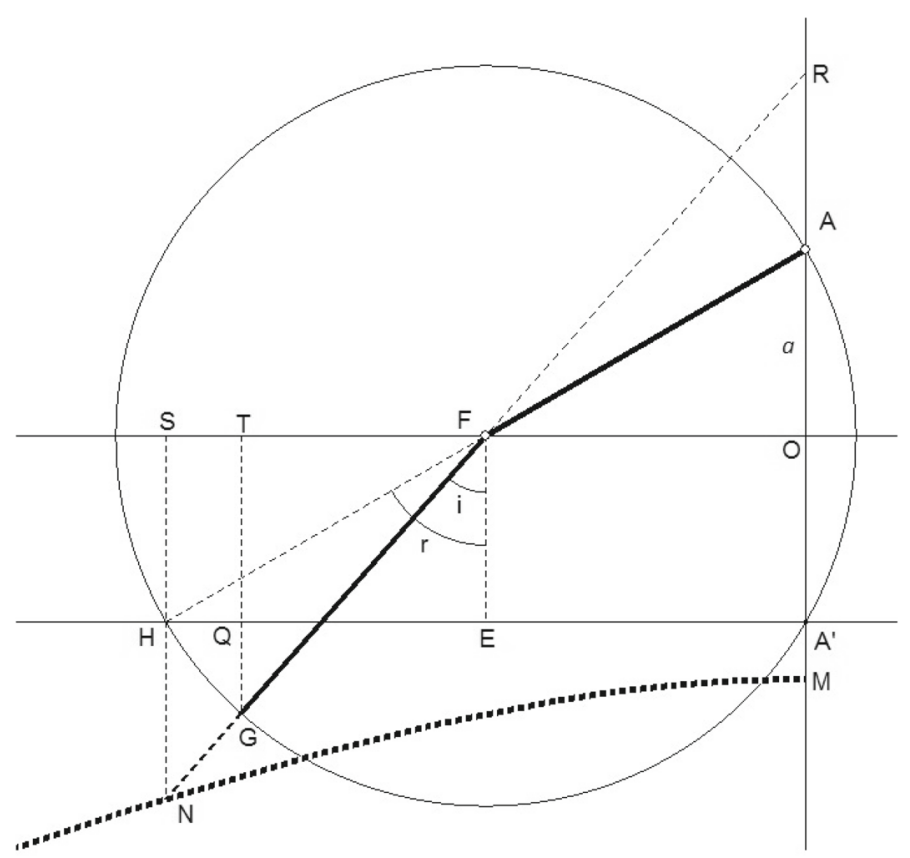

Fig. 11 Position of objects located under water as seen from $A$ at the same depth of $O A^{\prime}$

$$
\frac{H E}{Q E}=\frac{\sin r}{\sin i}=n_{12}
$$

These results show that the quotient that Heeffer anticipated is a co-exact attribute of the diagram as long as we do not demand the invariance of $R$. Thomas Harriot, in order to derive an approximate law to the law of sines, worked with a very similar diagram to the one we have suggested (Fig. 11; Schuster 2013, pp. 188-190).

This protocol can be used when the optical density of the second medium is less than that of the first medium (e.g, when light passes from water to air), i.e., when $n_{2}<n_{1}$ and $n_{21}<1$. In that case (and while admitting the reversibility of refraction), the calibration curve that is obtained for the new pair $\left(22.5^{\circ}, 30^{\circ}\right)$ is an ellipse and no longer a hyperbola (Fig. 12). Therefore, the parameter that we evaluated for the hyperbola $b_{\text {hyperbola }}^{2}=\frac{n_{21}+1}{n_{21}-1} a^{2}$ must be $b_{\text {ellipse }}^{2}=\frac{n_{21}+1}{1-n_{21}} a^{2}$ for $n_{21}<1$; therefore, the standard equation is transformed into an ellipse, i.e., $\frac{y^{2}}{a^{2}}+\frac{x^{2}}{b^{2}}=1$.

The heuristics do not encounter any obstacles as a result of this change because Kepler anticipated, in an insightful passage from the Paralipomena, the possibility of contemplating a unified theory of conics. In this passage, Kepler suggests that the parabola, which appears to be a transition between a hyperbola and an ellipse, is both the most acute of hyperbolas and the most acute of ellipses (See Kepler 1604/2000, IV, pp. 106-109, 1604/1939, pp. 90-94). 


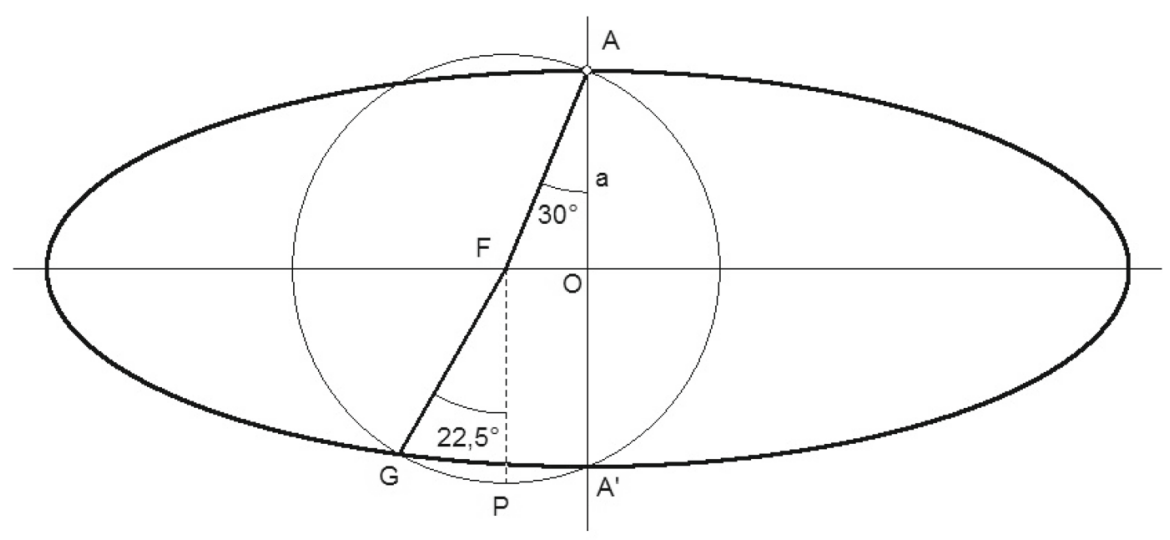

Fig. 12 Transition of light from a medium of lower optical density

If the second medium has the same optical density as the first medium, $n_{21}=1$, and the calibration curve then turns into a degenerated hyperbola; in other words, the curve turns into a pair of parallels to the horizontal axis. Kepler's unified theory of conics conceived a pair of parallel lines as being the most obtuse of hyperbolas and the circle as being the most obtuse of ellipses.

\section{The new model and the metaphysics of light}

Despite the failures, Kepler never gave up the hope of finding a hyperbola similar to the one that we have conceived in this article. Before he went on to develop the third family of analogies, Kepler encouraged his readers not to abandon this quest: "[w]hat will happen is that you find such a hyperbola, [...]; that is, that by this hyperbola [...] is contained the measure of all the refractions of water; and besides, that by other hyperbolas are contained the measures of media that are different with respect to refractions" (1604/2000, IV, p. 119, 1604/1939, p. 100). Now we must ask: What sort of metaphysical basis can we use to defend the model that we have constructed?

A scientist from the twenty-first century may be satisfied with a tool that anticipates the empirical results; initially, they may feel more comfortable in limiting their task to merely saving the phenomena. However, a thinker from the seventeenth century would not be happy at simply saving the phenomena. They would also demand a metaphysical basis that could account for the causal processes of the effects that can be anticipated when using the proposed model. One possible explanation consists of offering a mechanism that provides the reader with a number of efficient causes that can determine why nature behaves as it does, rather than behaving in other, alternative ways. In Kepler's defense of Tycho Brahe against Ursus with regard to the legal controversy between them, Kepler studied the role that hypothesis must play in scientific research. We may extend what he was trying to exemplify in astronomy to optics. Indeed, Kepler wrote: "we first depict the nature of things in hypothesis, then we construct out of them a method of calculation - that is, we demonstrate the motions" 
(1601/1988, p. 148). In this section, we would like to propose a plausible mechanism or hypothesis that could help us to conceive a successful method of calculation as the one we have presented.

Descartes deduced the law of sines by offering a mechanism that was closely related to the heuristics of the first family of analogies. Descartes acknowledged, in a letter from March 31, 1638, addressed to Marin Mersenne, the influence that Kepler had had on his inquiries on optics: "I recognize that Kepler was my first teacher in optics, and I think that he knew more about the subject than those who preceded him" (1897/1910, II, p. 86; personal translation). The model that we have described here came to our minds when we noticed the resemblance between Descartes' proposal and Kepler's heuristic recommendations. John Schuster asserts that Descartes carefully studied Kepler and took some of his recommendations (2013, pp. 195-197). Particularly, in his first approach to optics, the philosopher was inclined to consider light as the presence of an immaterial power that was perceived instantaneously without the transference of information with a finite velocity. ${ }^{30}$ Heeffer also suggests that Descartes arrived at the law of sines when he cautiously examined the diagram shown in Fig. 1 (2017, p. 151). Descartes presented the law in the first discourse of the Dioptrique. For its presentation, he offered three mechanical analogies. The first suggested that just as a blind man uses a walking stick to inform himself of the presence of external objects, light allows us to perceive the existence of objects located in front of us. Through this comparison, Descartes notes that just as the action that affects one of the ends of the walking stick reaches instantaneously the other end, light is also perceived instantaneously (Descartes 1897/1910, VI, 84). The second analogy compares the rectilinear propagation of the power of light with the transmission of the pressure from the upper layers to the lower layers in a vat full of grapes. The analogy demands that we make a distinction between the effective motion of different bunches of grapes and the tendency to move. A portion of matter cannot move through different paths, while the action attributed to light can. A light ray, conceived as a conceptual tool, does not represent the motion of a portion of matter, but the immediate expansion of an action (Descartes 1897/1910, VI, 86-88). Finally, when Descartes wanted to explain the effects of light when material objects are interposed, he compared the situation with balls that move and collide with each other or that cross a surface to enter a new medium. In these three analogies or cases, the phenomena associated with light are compared with mechanical events that are familiar to us. Now, it is not our goal to establish resemblances or differences between the manner in which Kepler and Descartes make use of analogies. We are now interested in presenting the argument that relied on the third comparison which Descartes offered in the Dioptrique to explain the law of sines.

Descartes asked the reader to imagine a ball that moves in the direction $A B$ (see Fig. 13). The surface $C B E$ represents the surface between two different media. The ball encounters a cloth $(C B E)$ that can be penetrated while absorbing some of the ball's speed. Let us say that the velocity after passing the cloth, denoted by $v_{2}$, is slowed down

\footnotetext{
30 Descartes was so convinced of the instantaneous expansion of the action of light, that in 1634 he wrote a letter, probably addressed to Isaac Beckman, where he said: "this is so true for me that, if anyone could prove its falsehood, I should be willing to confess that I know nothing of philosophy" (Descartes 1897/1910, I, p. 308).
} 


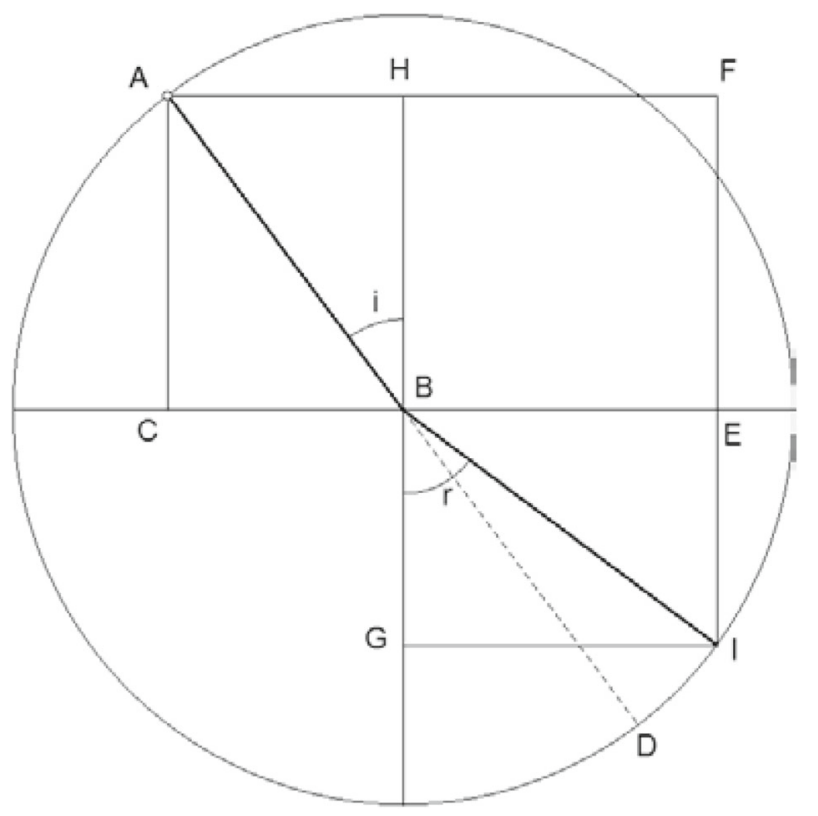

Fig. 13 Refraction (Cartesian mechanism)

by a factor of $n_{21}$, i.e., $v_{2}=n_{21} v_{1} .{ }^{31}$ The analysis requires the following working hypotheses (Descartes 1897/1910, VI, 93-105):

1. The motion prior to the encounter with the cloth along the path $A B$ can be decomposed into two independent motions: one motion in the direction $A C$ and the other in the direction $A H$. The real motion $A B$ can be obtained by adding these two components.

2. The encounter with the cloth slows down only one of these components, i.e., the motion in the direction $A C$; there is no reason to expect any change in the motion in the direction $A H$ :

3. After the intervention of the cloth, the total velocity of the ball is slowed down according to the relationship $v_{2}=n_{21} v_{1}$.

4. The velocity change occurs abruptly only when the object meets the cloth. ${ }^{32}$

To determine the new direction, Descartes drew a circle with center $B$ and radius $B A$. If, after passing the cloth, the ball's velocity is slowed down (or accelerated) by a factor $n_{21}$ (hypothesis 3), the time required to travel the distance $A B$ must be increased (or reduced) by a factor of $1 / n_{21}$. Therefore, when the ball moving from $B$ reaches any of the points on the circumference of the circle below the surface, the time would be increased (or reduced) by the factor $1 / n_{21}$. Given that the velocity in the direction $A H$ is not modified, (hypothesis 2), we must expect that in the time that has been increased

\footnotetext{
31 Descartes uses the factor $1 / 2$ in his presentation.

32 Mark Smith (1987, pp. 49-51) suggested that Descartes's analogy could have been motivated by an analogy proposed by Alhacen (trans. 2010). The Arab philosopher recommended to consider the cases of reflection and refraction of light as analogous to the encounter of material objects with thin barriers.
} 
(or reduced) by the factor $1 / n_{21}$, the distance traveled in that direction must increase (or decrease) by the same factor $1 / n_{21}$. For this reason, Descartes located the point $E$ on the surface such that $B E=\frac{1}{n_{21}} A H$. This quotient demands that $\frac{A H}{B E}=n_{21}$; hence, the quotient between the two segments replicates the quotient of the two velocities, which can simultaneously be read as an expression of the quotient of the two optical densities. Next, Descartes drew the perpendicular to $C B$ through $E$ and determined, on the sector that represents the new medium, the intersection $I$ with the circumference. When the two previous conditions are taken into consideration, it follows that the ball must take the direction $B I$. From this reasoning, it follows that $\frac{\sin i}{\sin r}=n_{21} \cdot{ }^{33}$ The close resemblance to the first group of analogies explored by Kepler immediately becomes obvious. $^{34}$

Descartes's analogy provoked many disagreements and has aroused many difficulties among scholars. On the one hand, the model implies that if the second medium is denser, then light propagates more easily. On the other hand, the model suggests two conflicting theses, that is, the instantaneous transmission of light and the variation in the velocity of light when it changes from one medium to another. Descartes was aware of the difficulty. In a letter to Mersenne he points out that "it seems that there is a particular disproportionality in that the motion of a ball is more or less violent, according as it is pushed with different forces, whereas light penetrates the diaphanous bodies in an instant and seems to have nothing successive in it" [quoted in Sabra (1981, p. 112)]. In several passages of the Dioptrique, Descartes insists that even though in the ball's case one talks about velocity, concerning light one must refer to the determination of the deployment of light, not to its velocity. John Schuster, in an effort to save Descartes from the incoherence, demands that we should keep in mind this distinction:

It is sometimes said Descartes fell into a contradiction, because in his theory of light, rays move instantaneously through any medium, whilst in the tennis ball model we must deal with a ratio of finite speeds. But, taking into account Descartes' dynamics and theory of light, we can now see that he had no problem: one must distinguish the speed of a light ray, which is instantaneous, from the magnitude of its force of propagation, which can take any positive value. The speed of Descartes' tennis ball corresponds not to the speed of propagation of light but to the intensity of the force of its propagation. (2013, p. 178)

\footnotetext{
33 Descartes does not use the expression $\sin$ in the presentation of his law, although it is clear that his reasoning leads to that presentation. He probably did not use the expression because it was not standardized in languages other than Latin. In fact, in a letter written to Mersenne (June 1632) Descartes presented the result in Latin, even though he had written the letter in French: "Pour la façon de mesurer les refractions de la lumiere, instituo comparationem inter sinus angulorum incidentiae \& angulorum refractorum" (Descartes 1897/1910, I, p. 255).

34 Huygens stated that he read a manuscript from Snell in which he stated the same result as Descartes. This manuscript, however, is not available, and so we must ignore the possibility that Snell got to the result using the same type of reasoning as Descartes. Sabra has considered the possible accusation of plagiarism that was leveled at Descartes; cfr. Sabra (1981, pp. 99-105). Fermat, who resisted admitting the validity of Descartes' demonstration because it introduced an unexpected result (i.e., that the velocity of light had to be faster in a denser medium), performed careful trials to derive the constancy of the quotient between the two sines, but from the relationship between the resistances offered by the media rather than focusing on the quotient of the velocities. For an analysis of these trials, see Sabra (1981, pp. 93-159).
} 


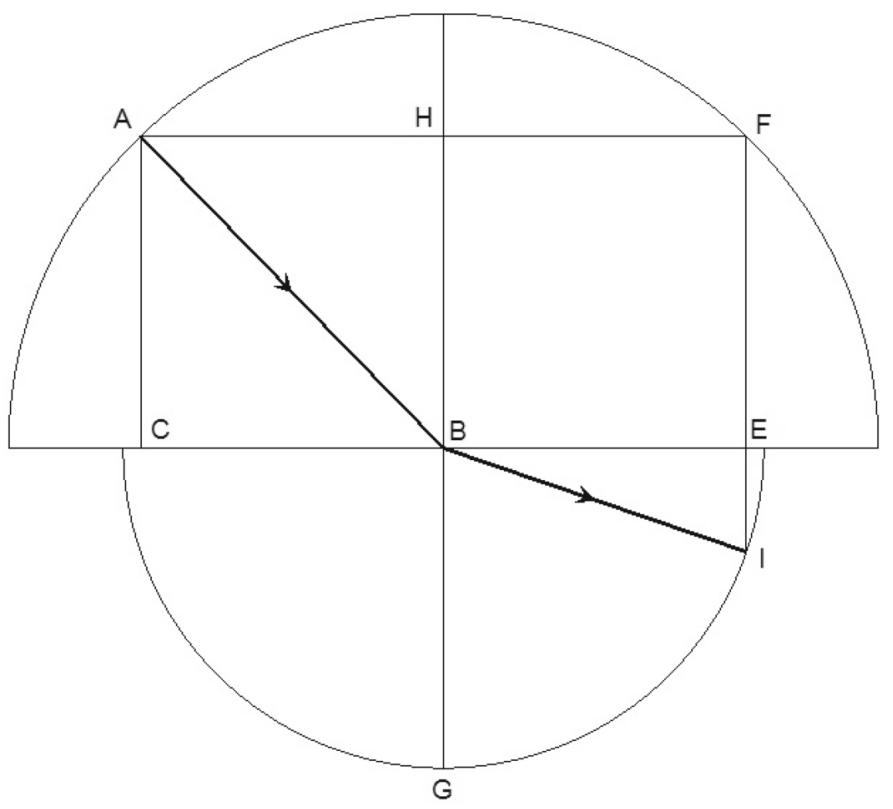

Fig. 14 Schuster's reinterpretation of Descartes

Schuster reinterpreted Descartes's proposal in order to avoid talking about the velocity of light in the derivation of the law of sines. The reasoning can be summarized in the following way. $A B$ represents the magnitude of the force of light's impulse (Fig. 14). This force of light, reduced by the factor $n_{21}$, is represented by the lower semicircle, whose radius has been reduced by the same factor. The figure shows that the parallel determination of the force has not been modified. The distance between $A C$ and $H B G$ stands for the parallel determination. This distance is equal to the separation between $H B G$ and $F E I$ that now represents the parallel determination of light after the change of medium. The intersection of the lower semicircle and the extension of $F E$ determines the new orientation of light and the magnitude of its force in the new medium.

We will not focus on interpreting what Descartes means by "action de la lumiére" (Descartes 1897/1910, VI, 100) nor we will evaluate whether Schuster's proposal saves Descartes from the incoherence or not. The matter we are interested in is whether Kepler, or a Keplerian philosopher, would accept either of the two versions of the Cartesian argument.

Why did Kepler not try a similar model if he was dealing with similar constructions? Kepler would not have accepted Descartes's first version of the analogy. While he would readily have admitted the possibility of the decomposition of the velocity of a moving physical object (Kepler 1604/2000, I, prop. 19, 20, pp. 27-29, 1604/1939, pp. 25-27), he would not have agreed with assigning a finite velocity to the propagation of light. We will now explore Kepler's reasons for resisting this suggestion. It is still necessary to ask whether a Keplerian philosopher would admit any version close to Schuster's reformulation. Now, we will address the principles of the metaphysics of light that were presented in the first chapter of the Paralipomena. 
In Kepler's defense of Tycho Brahe, he identified three important aspects of astronomy. We may identify, by analogy, the three aspects in optics. We will quote Kepler's passage and add in parenthesis the changes that will allow us to extend Kepler's recommendation to optics:

Altogether there are three things in astronomy [optics]: geometrical hypotheses; astronomical [optical] hypotheses; and the apparent motions of the stars themselves [the apparent behaviour of light]. Accordingly, there are two distinct tasks for an astronomer [perspectivist]: one, which truly pertains to astronomy [optics], is to set up astronomical [optical] hypotheses such that the apparent motions will follow from them [the behaviour of light will follow from them]; the other, which pertains to geometry, is to set up geometrical hypotheses of whatever kind such that from them those prior astronomical [optical] hypotheses, [...] both follow and can be worked out. (1601/1988, p.154)

At the moment, we have at hand the geometrical hypothesis that saves the phenomenon; the task now is to find the corresponding optical hypothesis. We will search for this hypothesis in Kepler's metaphysical presuppositions with regard to the nature of light.

As a result of the Neoplatonic influences that Kepler received at Tübingen, he recognized in the nature of all things the impulse to imitate God. ${ }^{35}$ Kepler used the figure of the Trinity to maintain that the nature and the spherical expansion of light were associated with the manifestation or the presence of God in the world: "Hence the point of the center is in a way the origin of the spherical solid, the surface the image of the inmost point, and the road to discovering it. The surface is understood as coming to be through an infinite outward movement of the point out of its own self" (Kepler 1604/2000, I, p. 19, 1604/1939, p. 19).

Light expresses the creative projection from the center to distant places. Light itself has no matter, weight or resistance (Kepler 1604/2000, I, prop. 3, p. 20, 1604/1939, p. 20). Light is considered as being the middle way between God the creator and the material world. For that reason, one can expect that the study of light will concur with geometrical laws (Kepler 1604/2000, I, prop. 2, p. 20, 1604/1939, p. 20). The straight lines that model the expansion of this creative force are called "rays," and the manifestation of this force is expressed in the spherical surface that has the source of light as its center (Kepler 1604/2000, I, prop. 4, p. 20, 1604/1939, p. 20). The expansion of light, given its divine nature, is instantaneous (Kepler 1604/2000, I, prop. 5 , p. 21, 1604/1939, p. 21). The rays, which were conceived as geometrical tools to model the unfolding of the creative force, are not part of the nature of light (Kepler $1604 / 2000$, I, prop. 8 , p. $22,1604 / 1939$, p. 21). In the same way that one can discern the difference between a straight line used to represent the motion of a physical body and the real motion of that body, which is not straight in any way, one can also, in the case of light, distinguish between the unfolding that occurs in a straight line and the unfolded nature of the light that acquires a spherical surface. The rays that represent the expansion of the light are perpendicular to the spherical surface that manifests this

\footnotetext{
35 To assess the importance of Neoplatonism in Kepler's education, the reader can consider the studies of Methuen (1998) and the article by Lindberg (1986).
} 
unfolding and creative power. ${ }^{36}$ Light thus has a two-dimensional nature. Therefore, given that only similar phenomena can have an effect on each other, there is no reason to expect that physical bodies, which are three-dimensional, will have an effect on light, which is two-dimensional (Kepler 1604/2000, I, prop. 10, p. 22, 1604/1939, p. 22). Given that approach, only the surface that embraces the physical medium, and not the inner or material structures, can influence the expansion of light (Kepler 1604/2000, I, prop. 13, 14, pp. 23-24, 1604/1939, p. 32). Therefore, a Keplerian philosopher would admit without any difficulty premise (4) from the Cartesian analogy.

In proposition 20 of the first chapter of the Paralipomena, Kepler proposed a description that allows the reader to understand why light that enters obliquely to a new medium with a greater optical density is then refracted towards the normal. We will present this description and will show how we can build a Keplerian defense for our model. Let $A$ be a light source, $H C$ be the surface between the two media, and let $B$ be the point of incidence of a ray coming from $A$; in addition, lines $B F$ and $A C E$ are perpendicular to $H C$ (Fig. 14). The new medium obstructs the expansion of light, not as a solid body, but as a surface that encloses the new medium which then encounters the spherical expansion of the light. The surface of this expansion meets the new medium's surface and forms an angle that is equivalent to the angle of incidence. A greater angle of incidence leads to an increase in the size of the shared surface component and, consequently, to a greater obstruction effect. ${ }^{37}$ The power emanating from $A$ that reaches the new medium at the angle of incidence is then distributed along the segment $C B$. If a denser medium were not in the way, all the power would then be distributed along the segment $E D$. Now, if the surface resists the expansion at a maximum without inhibiting the light from passing through it, the power that is distributed along the line $B C$ would only expand in the new medium along $E F$, which has the same dimensions as the segment $C B$. Therefore, the possible expansion in the new medium has two thresholds: $E F$ when the resistance is at maximum and $E D$ when the resistance is at a minimum. This reasoning allowed Kepler to conclude that "when a denser medium $B C$ comes in between, hindering the spreading out, it makes the light occupy a space that is intermediate between $E F$ and $E D$ : let this be $E G$. Therefore, the ray $A B$ will be refracted at $B$ and below the surface of the denser medium will become $B G$ ' (Kepler 1604/2000, I, prop. 20, p. 28, 1604/1939, pp. 26-27) (Fig. 15).

We will now use Kepler's suggestion to defend our tool (or model). $A B$ represents an incident ray that arrives at $B$ on the surface separating the two media where the second medium has a greater density than the first (Fig. 16). We will also assume that the surface resistance is dependent on the difference between the optical densities. Next, the power that is gathered at $B$ multiplies and expands over a new spherical surface with its center at $B$. Let us consider a sphere with radius $B A$. The ray $A B$ could expand in any of the directions between the two rays $B D$ and $B F$, as long as they remain perpendicular to the expanding sphere. When entering the second medium, the ray would follow a path between the maximum resistance and the minimum resistance that are produced by the touching surfaces. In other words, the ray would follow a

\footnotetext{
36 This is similar to the way in which wavefronts are perpendicular to the direction of propagation of the mechanical disturbance in an undulatory model.

37 This hypothesis is fundamental to the third family of analogies.
} 


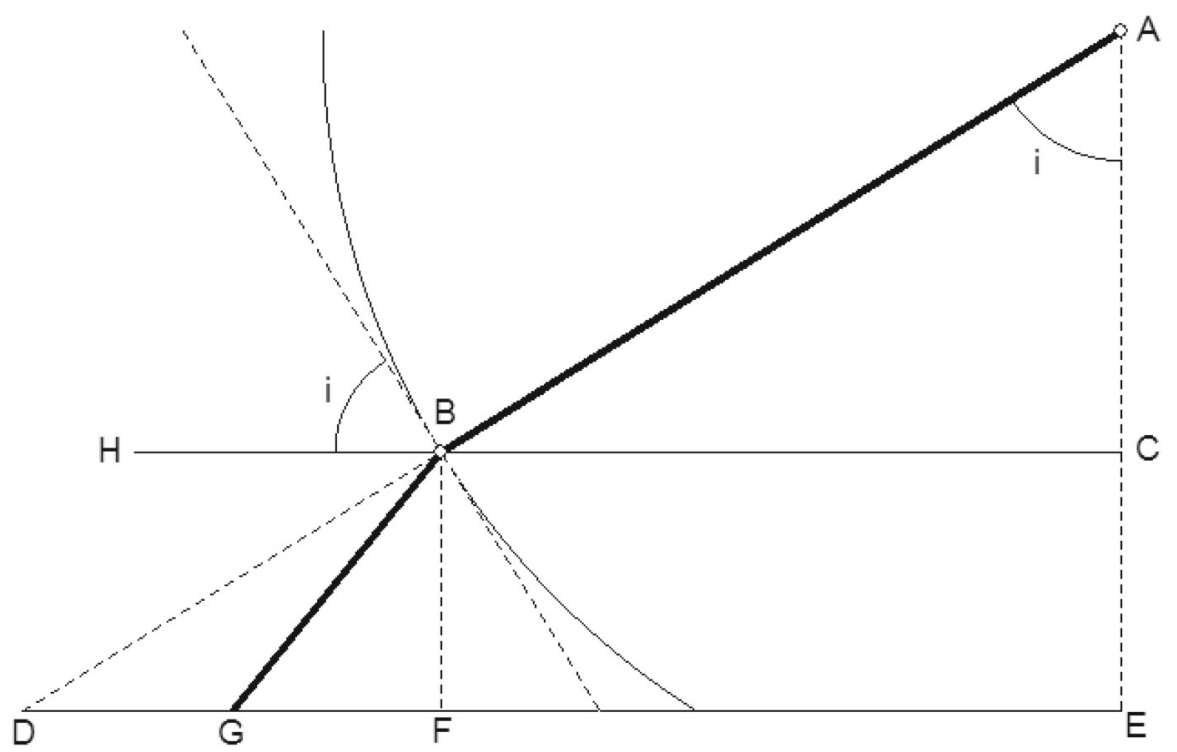

Fig. 15 Keplerian mechanism for the defense of the model

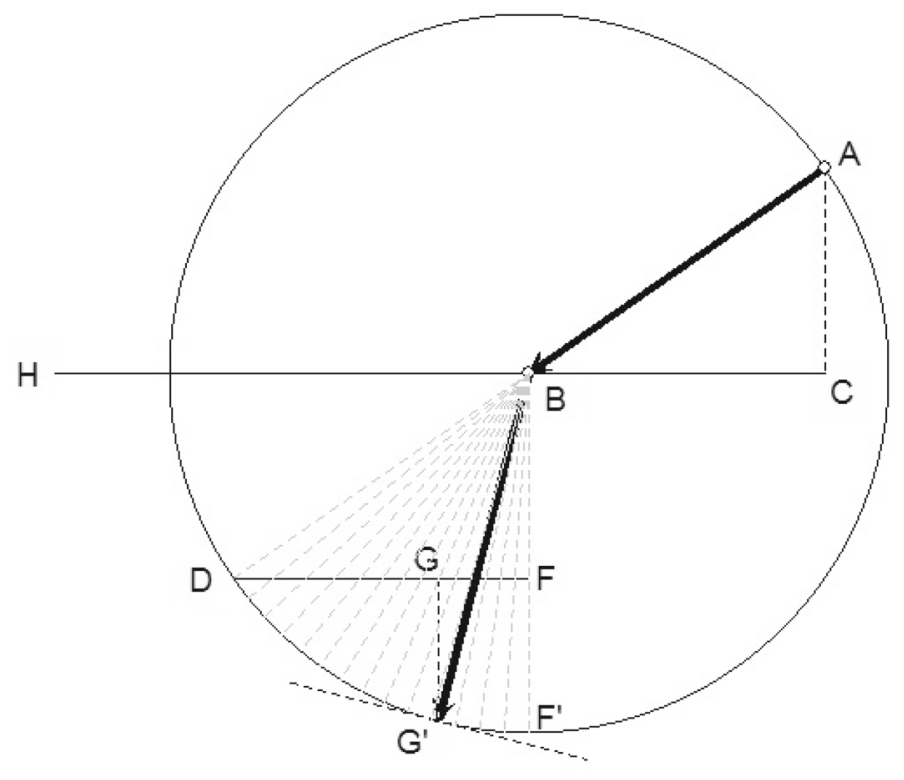

Fig. 16 Defense of the model

path between $D$ and $F$. The point $G$ is located on $D F$ such that $\frac{D F}{G F}=n_{21}$. Given that the power that is expanding from $B$ is distributed over a sphere and not on a plane, let us imagine that the power that is concentrated in $F G$ projects perpendicularly over the spherical surface on the sector $F^{\prime} G^{\prime}$. The new propagation direction concurs with the 
Table 3 Data from Thomas Harriot

\begin{tabular}{llllll}
\hline Weight & First medium & $\begin{array}{l}\text { Angle of } \\
\text { incidence } i\end{array}$ & $\begin{array}{l}\text { Angle of } \\
\text { refraction in air } r\end{array}$ & $\begin{array}{l}\text { Refractive index } \\
\text { of the medium } \\
\text { relative to air }\end{array}$ & $\begin{array}{l}\text { Refractive index } \\
\text { measured today }\end{array}$ \\
\hline 10 & Water & $30^{\circ}$ & $41.8^{\circ}$ & 1.3330 & 1.3333 \\
8.394 & Alcohol & $30^{\circ}$ & $43.5^{\circ}$ & 1.3767 & 1.36 \\
25.76 & Glass & $30^{\circ}$ & $51.066^{\circ}$ & 1.5557 & 1.5174 \\
10.744 & Amber & $30^{\circ}$ & $51.066^{\circ}$ & 1.5557 & 1.546 \\
\hline
\end{tabular}

The data of the first four columns are taken from Kepler, (1604-1611/2008, p. 312). Harriot gives the angle of deviation; we present, in decimal notation, the corresponding angle of refraction

direction indicated by the vector $B G^{\prime}$. Therefore, the light follows the path that our tool indicates. Unlike Descartes, Kepler expects that if the second medium is denser, then the resistance to the propagation of the power of light will be grater.

We now must consider the definition of the optical density of a medium. In 1606, Kepler began a correspondence with Thomas Harriot. This exchange lasted until 1609. In the first answer that Harriot sent to Kepler, he transcribed a table containing fifteen data sets that shows the behavior of light when passing from air into fifteen different media. Table 3 presents Harriot's data sets and the refractive index values that would be expected in each case according to the Snell-Descartes law. The table contains only four data sets, which are sufficient to illustrate the specific difficulties that Harriot presented to Kepler.

Harriot did not consider the angle of incidence in air, as Kepler and Witelo had done, but rather in the denser medium. The English scientist sent, for each medium, only the data that corresponded to a single angle of incidence: $30^{\circ}$. Despite Kepler's repeated demands, Harriot did not send further tables that contained the corresponding information for other angles of incidence. The comparison with the refractive indexes measured at present shows the agreement with the expected values deduced from the Snell-Descartes law. The most relevant aspect of the table is that it indicates that at first glance, there is no relevant correlation between the material densities of the media and the refraction. Contrary to what had been anticipated, when light passes from alcohol to air, a greater deviation is observed than when the light passes from water to air, despite the fact that alcohol is less dense. Similarly, glass and amber have very similar refractive behaviors with respect to the air, despite the fact that their densities differ substantially.

Kepler was surprised by the difference between water and alcohol and admitted that the optical density concept would have to differ from the material density concept. Kepler considered this problem as follows: "However, there is something hidden in the bodies, something that differs from the weight and that makes each one of them transparent; regarding this transparency, bodies are denser or less dense" (1604-1611/2008, pp. 321-322). Therefore, any metaphysical basis that could agree with our model and with Kepler's expectations must allude to the optical density as a concept that can be understood separately from material density and that has a nature that will have to be disentangled in future investigations. 


\section{Conclusions}

Kepler was unable to make the examplars of the first family or the second family anticipate the empirical results that were gathered in Witelo's table. However, he did not dismiss the possibility of constructing a representative hyperbola for each medium that could be used as an tool to predict the change in light's direction when passing from one medium to another. We have presented a way of constructing a hyperbola that agrees with the heuristics of the two families and that successfully embraces both Witelo's information and the expectations of the Snell-Descartes law. We have also accompanied our defense of this tool with the suggestion of a justification that would have harmonized with the metaphysics of light as conceived in the Paralipomena. This shows that Kepler's heuristics, the data he had at hand and his metaphysical presuppositions would have led him to the precise law of refraction that was incorporated by tradition. Therefore, if he was unable to formulate this law, it was not because his data were inaccurate, his metaphysics disorienting or his heuristics impracticable.

The third family of analogies led Kepler to a law of refraction that was later dismissed. This law guided Kepler to produce formidable results that were then adopted by tradition (e.g., the fact that a homocentric beam of light, when passing through the eye, converges later; the role of the retina in the reception of pictorial copies of external objects; the distinction between real images and virtual images, and between the images formed on the retina and those contemplated by the mind, among other things). Therefore, the influence of the Paralipomena on the consolidation of modern optics is undeniable. This influence is not restricted solely to the acceptance of the results mentioned above but can also be traced back to the heuristics of the investigation. We have seen that Descartes' arguments for conception of a law of refraction involve the influence of Kepler's methodology. Fermat's objections to Descartes, which led him to prioritize the role of the surface resistance of the new medium, and Huygens's distinction between waves and their directions of propagation have close resemblances to the methodological recommendations in Kepler's work.

Acknowledgements We thank David MacDonald, MSc, from Edanz Group (www.edanzediting.com/ac) for editing a draft of this manuscript.

Funding This work was funded by Universidad del Rosario, Bogotá, Colombia.

\section{Compliance with ethical standards}

Conflict of interest On behalf of all authors, the corresponding author states that there is no conflict of interest.

Open Access This article is distributed under the terms of the Creative Commons Attribution 4.0 International License (http://creativecommons.org/licenses/by/4.0/), which permits unrestricted use, distribution, and reproduction in any medium, provided you give appropriate credit to the original author(s) and the source, provide a link to the Creative Commons license, and indicate if changes were made. 


\section{References}

Alhacen. (trans. 2010). De Aspectibus (VII). Alhacen on Refraction, trans. and ed. Mark Smith. Philadelphia: American Philosophical Society.

Borrelli, Arianna. 2017. Optical Diagrams as "Paper Tools": Della Porta's Analysis of Biconvex Lenses from De refractione to De telescopio. In The Optics of Giambattista Della Porta (ca. 1535-1615): A Reassessment, ed. Arianna Borrelli, Giora Hon, and Yaakov Zik, 57-96. New York: Springer.

Buchdahl, Gerd. 1972. Methodological Aspects of Kepler's Theory of Refraction. Studies in History and Philosophy of Science 3: 265-298.

Cardona, Carlos. 2016a. Neopythagoreanism in the Work of Johannes Kepler. Manuscrito 39(3): 91-120.

Cardona, Carlos. 2016b. Kepler: Analogies in the Search for the law of refraction. Studies in History and Philosophy of Science 59: 22-35. https://doi.org/10.1016/j.shpsa.2016.05.004.

Caspar, Max. 1959/1993. Kepler. New York: Dover Publications Inc (Transl. by C. Doris Hellman).

de Fermat, Pierre. 1891. Synthesis ad Refractiones. In Euvres de Fermat, vol. I, eds. Paul Tannery and Charles Henry, pp 173-179. Paris: Impremerie Gauthier-Villars et fils.

Descartes, René. 1897/1910. Oeuvres de Descartes, eds. Charles Adam and Paul Tannery, 12 vols. Paris: Léopold Cerf.

Giardino, Valeria. 2017. Diagrammatic Reasoning in Mathematics. In Springer Handbook of Model Based Science, ed. Lorenzo Magnani and Tommaso Bertolotti, 499-521. New York: Springer.

Grant, Edward. 1974. A Source Book in Medieval Science. Cambridge: Harvard University Press.

Heeffer, Albrecht. 2014. Data-Driven Induction in Scientific Discovery: A Critical Assessment Based on Kepler's Discoveries. In Logic, Reasoning and Rationality, ed. E. Weber, D. Wouters, and J. Meheus, 59-76. Dordrecht: Springer.

Heeffer, Albrecht. 2017. Using invariances in Geometrical Diagrams: Della Porta, Kepler and Descartes on Refraction. In The Optics of Giambattista Della Porta (ca. 1535-1615): A Reassesment, eds. Arianna Borrelli, Giora Hon and Yaakov Zik, 145-168. New York: Springer.

Huygens, Christiaan. 1690/1945. Treatise on Light. Chicago: The University of Chicago Press (Transl. by Silvanus P. Thompson).

Itard, Jean. 1957. Les lois de la Refraction de la lumiere chez Kepler. Revue d'histoire des sciences et de leurs applications 10(1): 59-68.

Kepler, Johannes. 1601/1988. Apologia pro Tychone contra Ursus. In N. Jardine, The Birth of History and Philosophy of Science. Kepler's A Defence of Tycho Against Ursus with Essays on its Provenance and Significance, 83-207. Cambridge: Cambridge University Press.

Kepler, Johannes. 1604/2000. Optics: Paralipomena to Witelo \& Optical Part of Astronomy. Santa Fe: Green Lion Press (Transl. by William H. Donahue).

Kepler, Johannes. 1604/1939. Ad Vitellionem Paralipomena Quibus Astronomiae pars Optica traditur. In Gesammelte Werke $(G W)$, vol. II; München: C. H. Beck'sche Verlagsbuchhandlung; ed. and comm. Franz Hammer.

Kepler, Johannes. 1604-1611/2008. In Schriften zur Optik, ed. Rolf Riecher. Frankfurt: Verlag Harri Deutch. Kepler, Johannes. 1611/2008. Dioptrice. In Schriften zur Optik (1604-1611), ed. Rolf Riekher, 441-526. Frankfurt: Verlag Harri Deutsch.

Lindberg, David C. 1986. The Genesis of Kepler's Theory of Light: Light Metaphysics from Plotinus to Kepler. Osiris, Segunda Serie 2: 5-42.

Lohne, J. 1959. Thomas Harriot (1560-1621), the Tycho Brahe of Optics. Centaurus 6: 113-121.

Lohne, J.A. 1968. Der Eigenartige Einflu $\beta$ Witelos auf die Entwicklung der Dioptrik. Archive for History of Exact Sciences 4: 414-426.

Manders, Kenneth. 2008. The Euclidian Diagram. In The Philosophy of Mathematical Practice, ed. Paolo Mancosu, 80-133. New York: Oxford University Press.

Methuen, Charlotte. 1998. Kepler's Tübingen. Stimulus to a Theological Mathematics. Aldershot: Ashgate.

Risner, Frederich (ed.). 1572. Opticae Thesaurus Alhazeni Arabis libri septem... Item Vitellonis Thuring gopoloni libri decem. Basile: Per episcopios.

Sabra, A.I. 1981. Theories of Light from Descartes to Newton. Cambridge: Cambridge University Press.

Schuster, John. 2013. Descartes-Agonistes: Physico-mathematics, Method \& Corpuscular Mechanism. In Studies in History and Philosophy of Science, vol. 27, ed. Stephen Gaukroger. Dordrecht: Springer.

Smith, Mark. 1987. Descartes Theory of Light and Refraction: A Discourse on Method. Transactions of the American Philosophical Society 77(3): 1-92. 
Voelkel, James R. 2001. The Composition of Kepler's Astronomia Nova. Princeton: Princeton University Press.

Walker, D.P. 1978. Studies in Musical Science in the Late Renaissance. London: The Warburg Institute, University of London.

Zaiser, Hedwig. 1932. Kepler als Philosoph. Stuttgart: E. Suhrkamp.

Publisher's Note Springer Nature remains neutral with regard to jurisdictional claims in published maps and institutional affiliations. 